\title{
BALANCING DOMAIN DECOMPOSITION FOR MIXED FINITE ELEMENTS
}

\author{
LAWRENCE C. COWSAR, JAN MANDEL, AND MARY F. WHEELER
}

\begin{abstract}
The rate of convergence of the Balancing Domain Decomposition method applied to the mixed finite element discretization of second-order elliptic equations is analyzed. The Balancing Domain Decomposition method, introduced recently by Mandel, is a substructuring method that involves at each iteration the solution of a local problem with Dirichlet data, a local problem with Neumann data, and a "coarse grid" problem to propagate information globally and to insure the consistency of the Neumann problems. It is shown that the condition number grows at worst like the logarithm squared of the ratio of the subdomain size to the element size, in both two and three dimensions and for elements of arbitrary order. The bounds are uniform with respect to coefficient jumps of arbitrary size between subdomains. The key component of our analysis is the demonstration of an equivalence between the norm induced by the bilinear form on the interface and the $H^{1 / 2}$-norm of an interpolant of the boundary data. Computational results from a message-passing parallel implementation on an INTEL-Delta machine demonstrate the scalability properties of the method and show almost optimal linear observed speed-up for up to 64 processors.
\end{abstract}

\section{INTRODUCTION}

Balancing Domain Decomposition (BDD), introduced by Mandel in [24], is the further development of the methods proposed and studied by De Roeck and Le Tallec [17] based on an earlier work for the case of two subdomains [4]. The method involves the iterative solution by conjugate gradients of an interface problem preconditioned by the BDD preconditioner described in Algorithm 1 of $\S 5$ of this paper. The BDD algorithm involves at each iteration the solution of a local problem with Dirichlet data, a local problem with Neumann data, and a "coarse grid" problem to propagate information globally and to insure the consistency of the Neumann problems.

In this paper, we analyze the asymptotic convergence rate of the BDD method for the solution of mixed finite element discretizations of the following scalar, second-order, elliptic problem for unknown $p$ :

$$
\begin{array}{rlrl}
-\nabla \cdot A \nabla p & =f & & \text { in } \Omega, \\
p=g & & \text { on } \partial \Omega .
\end{array}
$$

Received by the editor March 18, 1993 and, in revised form, December 2, 1993.

1991 Mathematics Subject Classification. Primary 65N55.

Key words and phrases. Mixed finite elements, domain decomposition, parallel computing, second-order elliptic boundary value problems. 
We assume that $\Omega$ is a polygonal domain $\Omega \subset \mathbb{R}^{n}, n=2,3$, with boundary $\partial \Omega$. The matrix $A$ is symmetric and uniformly positive definite with bounded, measurable coefficients. The data $f$ and $g$ are assumed to be sufficiently regular, e.g., $f \in L^{2}(\Omega), g \in H^{\frac{1}{2}}(\partial \Omega)$. The choices of the polygonal domain and Dirichlet boundary conditions are for convenience; extensions to other cases are straightforward.

In mixed finite element approximations to (1.1)-(1.2) both the scalar variable, $p$, and its flux, $-A \nabla p$, are approximated. Used since the $1950 \mathrm{~s}$ in their earliest incarnation as cell-centered finite differences (cf. [31]), mixed finite element methods continue to be used in industrial problems (e.g. [18]) because of their inherent mass conservation properties and their high-quality approximation of both the scalar variable and its flux.

As is well known, the mixed formulation gives rise to a saddle point problem that is symmetric but indefinite. The BDD method, as well as many other effective domain decomposition techniques, is best suited for symmetric positive definite systems. In [22], Glowinski and Wheeler formulated the first domain decomposition methods for mixed finite elements. The two methods proposed in [22], denoted "Method I" and "Method II", are both substructuring methods that lead to symmetric, positive definite bilinear forms involving the unknowns on the interface.

In this paper, we apply the BDD preconditioner from [24, 25] to the "Method II" of Glowinski and Wheeler. The role of the subdomain Schur complement and its pseudoinverse from [24, 25] are played by the appropriate mixed finite element version of the "Dirichlet to Neumann" and "Neumann to Dirichlet" maps. Since all the BDD method requires are subroutines implementing those maps, the details of mixed finite elements and the fact that the problem is indefinite are hidden from the BDD algorithm itself. The resulting algorithm can be naturally interpreted as preconditioning "Method II" by "Method I".

The main theoretical result of this paper is the proof that the condition number of the preconditioned system grows at worst like $O\left(\left(1+\log \left(\frac{H}{h}\right)\right)^{2}\right)$ in both two and three dimensions and for elements of any order, where $H$ is the characteristic subdomain size and $h$ the characteristic mesh size. This is the same bound demonstrated for the application of the BDD method to a standard conforming Galerkin finite element method analyzed in [25]. In essence, the bounds on the condition number presented here work by reducing the mixed domain decomposed problem to a related conforming problem and applying the results of [25]. The key component of our analysis is the demonstration of an equivalence between the norm induced by the bilinear form on the interface and the $H^{1 / 2}$-norm of an interpolant of the boundary data. Using similar techniques, Cowsar [15, 14] has obtained an optimal analysis for the classical overlapping Schwarz methods and results for other substructuring Schwarz methods applied to a mixed finite element formulation with interelement multipliers.

The remainder of this paper is divided into six additional sections. In the next section, we introduce some notations and other preliminaries. For completeness, we discuss the mixed finite element method in $\S 3$. Readers familiar with the interelement multiplier formulation of the mixed method 
can simply skim this section to set some notation. In the fourth section, we formulate the equivalent problem in terms of the dual variables and analyze the resulting quadratic form. The dual problem is reduced to an interface problem in $\S 5$ and the BDD preconditioner is introduced. In $\S 6$, the bound on the condition number is derived. The paper concludes with some numerical results from a distributed memory message-passing implementation of the algorithm on the INTEL-Delta parallel computer, which confirm the theoretical bound and investigate the algorithm's parallel performance.

\section{NOTATION AND PRELIMINARIES}

Let $\mathscr{T}$ be a quasi-regular "triangulation" of $\Omega$ with characteristic mesh parameter $h$. The elements of $\mathscr{T}$ are not limited to triangles (tetrahedra in 3-D), but may, more generally, include various types of convex polygons. Let the domain $\Omega$ be subdivided into nonoverlapping subdomains $\Omega_{1}, \ldots, \Omega_{k}$, each of which is the union of elements of $\mathscr{T}$. We assume the subdomains $\Omega_{i}$ are of diameter $O(H)$ and shape regular; that is, there exist bijections $F_{i}$ from a reference domain of diameter $O(1)$ (e.g., a square in 2D or a cube in 3D) onto $\Omega_{i}$ with the Jacobian $D F_{i}$ satisfying the following estimates uniformly in $x$ :

$$
\left\|D F_{i}(x)\right\| \leq C H, \quad\left\|D F_{i}^{-1}(x)\right\| \leq C H^{-1} .
$$

Here, and throughout this paper, $C$ will denote a generic constant not necessarily the same from line to line, but always independent of mesh parameters $h$ and $H$.

We decompose the tensor $A$ in (1.1) as

$$
A(x)=\alpha(x) \widehat{A}(x),
$$

where $\alpha$ is a positive function that is piecewise constant on each subdomain with value $\alpha_{i}$ on $\Omega_{i}$. The uniform positivity of $A$ then implies the existence of constants $C_{1}, C_{2}>0$ such that the following bounds hold:

$$
C_{1} \alpha_{i} \xi^{T} \xi \leq \xi^{T} A(x) \xi \leq C_{2} \alpha_{i} \xi^{T} \xi \quad \forall \xi \in \mathbb{R}^{n}, \forall x \in \Omega_{i} .
$$

Let $d x$ denote the standard Lebesgue $n$-dimensional measure and $d s$ the $(n-1)$-dimensional surface measure. For a bounded open set $\omega \subseteq \mathbb{R}^{n}$, let $|\omega|$ denote the measure of the set, and $L^{2}(\omega),\left(L^{2}(\omega)\right)^{n}, H^{s}(\omega),\left(H^{s}(\omega)\right)^{n}$ denote the standard Sobolev spaces of real-valued functions defined on $\omega$. (See, e.g., $[1,23])$. Likewise, let $L^{2}(\partial \omega)$ and $H^{s}(\partial \omega)$ denote the usual Sobolev spaces defined on $\partial \omega$. Let $\mathbf{H}\left(\omega\right.$; div) be the subspace of $\left(L^{2}(\omega)\right)^{n}$ of functions with divergences in $L^{2}(\omega)$, i.e.,

$$
\mathbf{H}(\omega ; \operatorname{div})=\left\{\mathbf{v} \in\left(L^{2}(\omega)\right)^{n} \mid \nabla \cdot \mathbf{v} \in L^{2}(\omega)\right\} .
$$

Define the scaled Sobolev norms

$$
\|u\|_{1, \Omega_{i}}^{2}=|u|_{1, \Omega_{i}}^{2}+\frac{1}{H^{2}}\|u\|_{0, \Omega_{i}}^{2}, \quad\|u\|_{1 / 2, \partial \Omega_{i}}^{2}=|u|_{1 / 2, \partial \Omega_{i}}^{2}+\frac{1}{H}\|u\|_{0, \partial \Omega_{i}}^{2},
$$


where

$$
\begin{gathered}
\|u\|_{0, \Omega_{i}}^{2}=\int_{\Omega_{i}}|u(x)|^{2} d x, \quad\|u\|_{0, \partial \Omega_{i}}^{2}=\int_{\partial \Omega_{i}}|u(s)|^{2} d s, \\
|u|_{1, \Omega_{i}}^{2}=\int_{\Omega_{i}}|\nabla u(x)|^{2} d x, \quad|u|_{1 / 2, \partial \Omega_{i}}^{2}=\int_{\partial \Omega_{i}} \int_{\partial \Omega_{i}} \frac{|u(t)-u(s)|^{2}}{|t-s|^{n}} d t d s .
\end{gathered}
$$

We need to define local subspaces derived from a parent space. In general, having defined a parent space of functions $\mathscr{X}(\Omega)$ and a set $\omega \subset \Omega$, we will adopt the notation $\mathscr{X}(\omega)$ for the restriction of $\mathscr{X}(\Omega)$ to $\omega$, i.e.,

$$
\mathscr{X}(\omega)=\left\{\phi_{\mid \omega} \mid \phi \in \mathscr{X}(\Omega)\right\}
$$

We say that two quadratic forms $\mathscr{Q}_{1}$ and $\mathscr{Q}_{2}$ with the same domain $\mathscr{D}$ are equivalent if there exist constants $c, C>0$ such that

$$
c \mathscr{Q}_{1}(\phi, \phi) \leq \mathscr{Q}_{2}(\phi, \phi) \leq C \mathscr{Q}_{1}(\phi, \phi), \quad \forall \phi \in \mathscr{D},
$$

and write

$$
\mathscr{Q}_{1} \simeq \mathscr{Q}_{2}
$$

In what follows, the constants that appear in the equivalences are independent of $h, H$ and $\alpha_{i}$, but may depend on the constants in (2.3), (2.1), the degree of the mixed finite elements, and the regularity of the triangulation.

\section{MiXed AND INTERELEMENT MULTIPLIER FORMULATION}

In this section, we formulate the mixed finite element approximation for (1.1)-(1.2) and an equivalent hybrid form by introducing interelement multipliers. To that end, we note that we may rewrite (1.1) as the first-order system

$$
\begin{aligned}
A^{-1} \mathbf{u}+\nabla p & =0 & & \text { in } \Omega, \\
\nabla \cdot \mathbf{u} & =f & & \text { in } \Omega .
\end{aligned}
$$

Multiplying by appropriate test functions, integrating (3.1) by parts and using the boundary condition (1.2), we arrive at the following weak form: Find $\mathbf{u} \in \mathbf{H}(\Omega ;$ div $)$ and $p \in L^{2}(\Omega)$ such that

$$
\begin{gathered}
\int_{\Omega} A^{-1} \mathbf{u} \cdot \mathbf{v} d x-\int_{\Omega} p \nabla \cdot \mathbf{v} d x=-\int_{\partial \Omega} g \mathbf{v} \cdot \nu d s \quad \forall \mathbf{v} \in \mathbf{H}(\Omega ; \operatorname{div}), \\
\int_{\Omega} \nabla \cdot \mathbf{u} w d x=\int_{\Omega} f w d x \quad \forall w \in L^{2}(\Omega) .
\end{gathered}
$$

Let $\mathbf{V}_{h}(\Omega)$ and $W_{h}(\Omega)$ be finite-dimensional subspaces of $\mathbf{H}(\Omega$; div $)$ and $L^{2}(\Omega)$, respectively, defined on the triangulation $\mathscr{T}$. By the mixed finite element approximation to (3.3)-(3.4), we mean the pair $\left\{\mathbf{u}_{h}, p_{h}\right\} \in \mathbf{V}_{h}(\Omega) \times$ 
$W_{h}(\Omega)$ satisfying

$$
\begin{aligned}
\int_{\Omega} A^{-1} \mathbf{u}_{h} \cdot \mathbf{v} d x- & \int_{\Omega} p_{h} \nabla \cdot \mathbf{v} d x=-\int_{\partial \Omega} g \mathbf{v} \cdot \nu d s \quad \forall \mathbf{v} \in \mathbf{V}_{h}(\Omega), \\
\int_{\Omega} \nabla \cdot \mathbf{u}_{h} w d x & =\int_{\Omega} f w d x \quad \forall w \in W_{h}(\Omega) .
\end{aligned}
$$

For (3.5)-(3.6) to be well posed, the spaces $V_{h}(\Omega)$ and $W_{h}(\Omega)$ cannot be chosen independently. A well-known sufficiency condition is the LadyzhenskayaBabuška-Brezzi inf-sup condition [8]. There are many mixed finite element spaces defined in the literature for both two and three dimensions that satisfy this condition. These include the Raviart-Thomas-Nedelec spaces [28, 27], the BDM spaces [11,9], and the BDFM spaces [10]. The analysis presented in the subsequent sections shall be applicable to all of these spaces.

The mixed finite element spaces listed above admit an element-wise construction. In particular, they may be defined by two fixed finite-dimensional spaces $W_{h}(\tilde{\tau}) \subset L^{2}(\tilde{\tau})$ and $\mathbf{V}_{h}(\tilde{\tau}) \subset \mathbf{H}(\tilde{\tau}$; div) on a fixed reference element $\tilde{\tau}$ and a family of bijective affine maps to the elements in the triangulation $\mathscr{T}$,

$$
\left\{F_{\tau} \equiv b_{\tau}+B_{\tau} \tilde{x}: \tilde{\tau} \rightarrow \tau, \tau \in \mathscr{T}\right\}
$$

in the following way. Let

$$
\begin{gathered}
W_{h}(\tau)=\left\{p \mid p=\tilde{p} \circ F_{\tau}^{-1}, \tilde{p} \in W_{h}(\tilde{\tau})\right\} \\
\mathbf{V}_{h}(\tau)=\left\{\left.\mathbf{v}|\mathbf{v}=| \operatorname{det} B_{\tau}\right|^{-1} B_{\tau} \widetilde{\mathbf{v}} \circ F_{\tau}^{-1}, \widetilde{\mathbf{v}} \in \mathbf{V}_{h}(\tilde{\tau})\right\}
\end{gathered}
$$

and set

$$
W_{h}(\Omega)=\bigoplus_{\tau \in \mathscr{T}} W_{h}(\tau), \quad \mathbf{V}_{h}^{-1}(\Omega) \equiv \bigoplus_{\tau \in \mathscr{T}} \mathbf{V}_{h}(\tau) .
$$

Let $\mathbf{V}_{h}(\Omega)$ be the subset of $\mathbf{V}_{h}^{-1}(\Omega)$ composed of functions such that the normal component is continuous across the boundaries of elements. The continuity of the normal component and the fact that $\mathbf{V}_{h}(\tau) \subset \mathbf{H}(\tau$; div) insure that $\mathbf{V}_{h}(\Omega) \subset \mathbf{H}(\Omega ; \operatorname{div})$.

Recall that the above referenced spaces also have the property that

$$
\operatorname{div}\left(\mathbf{V}_{h}(\Omega)\right) \subseteq W_{h}(\Omega) .
$$

Also for each of these mixed finite element spaces, there exists a projection

$$
\Pi_{h}: \mathbf{H}(\Omega ; \operatorname{div}) \cap\left\{\mathbf{v} \cdot \nu_{\tau} \in L^{2}(\partial \tau), \tau \in \mathscr{T}\right\} \rightarrow \mathbf{V}_{h}(\Omega)
$$

that satisfies, among other properties, that for every $\tau \in \mathscr{T}$ and edge (face in 3-D) $e_{i}$ of the boundary of $\tau$

$$
\begin{aligned}
\int_{e_{i}}\left(\Pi_{h} \mathbf{u}-\mathbf{u}\right) \cdot \nu_{\tau} \mathbf{v} \cdot \nu_{\tau} d s=0 & \forall \mathbf{v} \in \mathbf{V}_{h}(\tau), \\
\int_{\tau} \nabla \cdot\left(\Pi_{h} \mathbf{u}-\mathbf{u}\right) q d x=0 & \forall q \in W_{h}(\tau) .
\end{aligned}
$$

In the presentation of the algorithm and its subsequent analysis, it will be convenient to present an equivalent form of the mixed problem by introducing Lagrange multipliers to enforce the continuity of the normal component of $\mathbf{u}$ 
between elements. As in [22], an implementation needs to use the interelement multipliers only on the interfaces of the subdomains, where they play the role of the trace of $p$. Let $\Lambda_{h}(\Omega)$ be the space of traces of the normal component of the flux on the element boundaries; that is,

$$
\Lambda_{h}(\Omega)=\bigcup_{\tau \in \mathscr{T}}\left\{\mathbf{v} \cdot \nu_{\tau} \mid \mathbf{v} \in \mathbf{V}_{h}(\tau)\right\}
$$

For $g \in L^{2}(\partial \Omega)$, let $\Lambda_{h}^{g}(\Omega)$ be the subset of all functions from $\Lambda_{h}(\Omega)$ which attain the boundary value $g$ on $\partial \Omega$ in a weak sense,

$$
\Lambda_{h}^{g}(\Omega)=\left\{\lambda \in \Lambda_{h}(\Omega) \mid \int_{\partial \Omega}(\lambda-g) \mu d s=0, \forall \mu \in \Lambda_{h}(\Omega)\right\} .
$$

The mixed problem (3.5)-(3.6) may be reformulated as finding the triple

$$
\left\{\mathbf{u}_{h}, p_{h}, \lambda_{h}\right\} \in \mathbf{V}_{h}^{-1}(\Omega) \times W_{h}(\Omega) \times \Lambda_{h}^{g}(\Omega)
$$

satisfying for all $\{\mathbf{v}, q, \mu\} \in \mathbf{V}_{h}^{-1}(\Omega) \times W_{h}(\Omega) \times \Lambda_{h}^{0}(\Omega)$ the equations

$$
\begin{gathered}
\sum_{\tau \in \mathscr{T}}\left(\int_{\tau} A^{-1} \mathbf{u}_{h} \cdot \mathbf{v} d x-\int_{\tau} p_{h} \nabla \cdot \mathbf{v} d x+\int_{\partial \tau} \lambda_{h} \mathbf{v} \cdot \nu_{\tau} d s\right)=0, \\
\sum_{\tau \in \mathscr{F}}-\int_{\tau} q \nabla \cdot \mathbf{u}_{h} d x=-\int_{\Omega} f q d x, \\
\sum_{\tau \in \mathscr{T}} \int_{\partial \tau} \mu \mathbf{u}_{h} \cdot \nu_{\tau} d s=0 .
\end{gathered}
$$

The additional function $\lambda_{h}$ admits a simple and important interpretation. If we consider the constitutive relationship (3.1) on a single element $\tau$, then. after multiplying by a test function and integrating by parts, we find that

$$
\int_{\tau} A^{-1} \mathbf{u} \cdot \mathbf{v} d x-\int_{\tau} p \nabla \cdot \mathbf{v} d x+\int_{\partial \tau} p \mathbf{v} \cdot \nu_{\tau} d s=0
$$

Comparing this with (3.9), we see that $\lambda_{h}$ is naturally interpreted as an approximation to the trace of $p$ on the boundaries of the elements.

Note that (3.11) imposes the continuity condition on the normal component of the flux that guarantees that $\mathbf{u}_{h}$ is in $\mathbf{V}_{h}(\Omega)$. The equivalence of (3.9)(3.11) and (3.5)-(3.6) follows essentially from this fact. See [2] for a proof of the equivalence and more information concerning this interelement multiplier formulation.

\section{The DUAL PROBLEM}

Henceforth, we shall only be concerned with the solution of the finitedimensional problem (3.9)-(3.11) and will consequently drop the subscript $h$ from $\mathbf{u}_{h}, p_{h}$ and $\lambda_{h}$. We parameterize the space $W_{h}(\Omega)$ elementwise by using a local nodal basis with nodes in the interior of elements. Likewise, we parameterize $\Lambda_{h}(\Omega)$ by a nodal basis defined on the edges (faces) of elements of the triangulation. We denote the discrete space of nodal values 
of $W_{h}(\Omega) \times \Lambda_{h}(\Omega)$ by $\mathscr{P}(\Omega)$. Because of $(3.12), \mathscr{P}(\Omega)$ has the natural interpretation as the space of values of the scalar variable $p$ on all nodes, in the interior as well as on the edges (faces in $3 D$ ) of the elements. Since $\mathscr{P}(\Omega)$ is isomorphic to $W_{h}(\Omega) \times \Lambda_{h}(\Omega)$ by construction, we may write (by abuse of notation) $\hat{p}=[p, \lambda]$, and consider $[p, \lambda]$ elements of $\mathscr{P}(\Omega)$.

In a variational framework, one may eliminate the flux variable $\mathbf{u}$ in (3.9)(3.11) by introducing a discretization of the flux operator $A \nabla$ denoted

$$
\nabla_{h}^{A}: W_{h}(\Omega) \times \Lambda_{h}(\Omega) \rightarrow \mathbf{V}_{h}^{-1}(\Omega),
$$

and defined by

$$
\sum_{\tau \in \mathscr{T}} \int_{\tau} A^{-1} \nabla_{h}^{A}[q, \mu] \cdot \mathbf{v} d x=\sum_{\tau \in \mathscr{T}}\left(-\int_{\tau} q \nabla \cdot \mathbf{v} d x+\int_{\partial \tau} \mu \mathbf{v} \cdot \nu_{\tau} d s\right) \quad \forall \mathbf{v} \in \mathbf{V}_{h}^{-1}(\Omega) .
$$

Since $\mathbf{V}_{h}^{-1}(\Omega)$ is the direct sum of spaces defined on each element, we note that (4.1) holds element by element. Hence, $\nabla_{h}^{A}[q, \mu]$ is defined elementwise in terms of the values of $q$ and $\mu$ restricted to $\bar{\tau}$. Therefore, by an abuse of notation, we may consider $\nabla_{h}^{A}$ as a map from $W_{h}(\omega) \times \Lambda_{h}(\omega)$ into $V_{h}^{-1}(\omega)$ for any set $\omega \subset \Omega$ which is the union of elements of $\mathscr{T}$.

Let

$$
d_{\Omega}([p, \lambda],[q, \mu])=\sum_{\tau \in \mathcal{F}} \int_{\tau} A^{-1} \nabla_{h}^{A}[p, \lambda] \cdot \nabla_{h}^{A}[q, \mu] d x .
$$

More generally, by recognizing the element-by-element nature of $d_{\Omega}(\cdot, \cdot)$, define

$$
d_{\omega}([p, \lambda],[q, \mu])=\sum_{\tau \in \mathcal{F}, \tau \subset \omega} \int_{\tau} A^{-1} \nabla_{h}^{A}[p, \lambda] \cdot \nabla_{h}^{A}[q, \mu] d x
$$

for $\omega \subset \Omega$ composed of elements of the triangulation $\mathscr{T}$. A problem equivalent to (3.9)-(3.11) is finding the pair

$$
[p, \lambda] \in W_{h}(\Omega) \times \Lambda_{h}^{g}(\Omega)
$$

satisfying

$$
d_{\Omega}([p, \lambda],[q, \mu])=\int_{\Omega} f q d x \quad \forall[q, \mu] \in W_{h}(\Omega) \times \Lambda_{h}^{0}(\Omega) .
$$

The flux may be recovered by $\mathbf{u}=-\nabla_{h}^{A}[p, \lambda]$. The bilinear form $d_{\Omega}(\cdot, \cdot)$ is obviously positive semidefinite. The strict positivity is a simple corollary to Theorem 4.1 below. A detailed discussion is given for the case of the RaviartThomas elements in [2] and [7].

The following theorem is the main result of this section. It spells out the key observation that the quadratic form $d_{\omega}(\widehat{p}, \widehat{p})$ is equivalent to a discretization of the form $\int_{\omega} \alpha|\nabla p|^{2} d x$, which will allow us to use the theory for the conforming case with only a few changes. 
Theorem 4.1. Let $\widehat{p}=[p, \lambda] \in W_{h}(\omega) \times \Lambda_{h}(\omega)$ for $\omega \subseteq \Omega$ composed of elements of the triangulation $\mathscr{T}$, and $\alpha_{\tau}=\alpha_{\mid \tau}$; then

$$
d_{\omega}(\hat{p}, \hat{p}) \simeq \sum_{\tau \in \mathscr{T}, \tau \subset \omega} \alpha_{\tau}|\tau|^{1-2 / n} \sum_{\substack{\text { nodes : } \\ n_{i}, n_{j} \in \tau}}\left(\widehat{p}_{\tau}\left(n_{i}\right)-\widehat{p}_{\tau}\left(n_{j}\right)\right)^{2}
$$

with constants independent of $h, \alpha$, and $|\omega|$.

The proof of Theorem 4.1 is a direct consequence of the following two lemmas.

Lemma 4.2. The kernel of $d_{\tau}(\cdot, \cdot)$ consists of the constant functions on $\bar{\tau}$, i.e.,

$$
d_{\tau}([p, \lambda],[q, \mu])=0 \quad \forall[q, \mu] \in W_{h}(\tau) \times \Lambda_{h}(\tau),
$$

if, and only if, $[p, \lambda]$ has the same values on all nodes of $\bar{\tau}$.

Proof. We first check that if $[p, \lambda]$ is some constant $K$ on $\bar{\tau}$, then it is in the kernel of $d_{\tau}(\cdot, \cdot)$. Letting $\mathbf{v}=\nabla_{h}^{A}[q, \mu] \in \mathbf{V}_{h}(\tau)$, we see that

$$
\begin{aligned}
d_{\tau}([p, \lambda],[q, \mu]) & =\int_{\tau} A^{-1} \nabla_{h}^{A}[p, \lambda] \cdot \mathbf{v} d x \\
& =-\int_{\tau} p \nabla \cdot \mathbf{v} d x+\int_{\partial \tau} \lambda \mathbf{v} \cdot \nu_{\tau} \\
& =K\left[-\int_{\tau} \nabla \cdot \mathbf{v} d x+\int_{\partial \tau} \mathbf{v} \cdot \nu d s\right],
\end{aligned}
$$

which is zero by the Divergence Theorem.

To prove the converse, let $[\tilde{p}, \tilde{\lambda}] \in W_{h}(\tau) \times \Lambda_{h}(\tau)$ be such that

$$
d_{\tau}([\tilde{p}, \tilde{\lambda}],[q, \mu])=0 \quad \forall[q, \mu] \in W_{h}(\tau) \times \Lambda_{h}(\tau) .
$$

It is enough to show that $[\tilde{p}, \tilde{\lambda}]$ is zero if it is orthogonal to constants, i.e.,

$$
\int_{\tau} K \tilde{p} d x+\int_{\partial \tau} K \tilde{\lambda} d s=0 \quad \forall K \in \mathbb{R} .
$$

By (4.6),

$$
\int_{\tau} A^{-1} \nabla_{h}^{A}[\tilde{p}, \tilde{\lambda}] \cdot \nabla_{h}^{A}[\tilde{p}, \tilde{\lambda}] d x=0
$$

and hence,

$$
\nabla_{h}^{A}[\tilde{p}, \tilde{\lambda}]=0 .
$$

Since (4.7) holds, there exists a solution $\phi$ (unique up to a constant) satisfying the following Neumann problem:

$$
\begin{array}{rlrl}
-\Delta \phi=\tilde{p} & \text { in } \tau, \\
\nabla \phi \cdot \nu=\tilde{\lambda} & & \text { on } \partial \tau .
\end{array}
$$


Setting $\tilde{\mathbf{v}}=\Pi_{h} \nabla \phi$ and using properties (3.7) and (3.8), we see that $\tilde{\mathbf{v}} \in \mathbf{V}_{h}(\tilde{\xi})$ with $\nabla \cdot \tilde{\mathbf{v}}=-\tilde{p}$ and $\tilde{\mathbf{v}} \cdot \nu_{\tau}=\tilde{\lambda}$. Hence, using $\tilde{\mathbf{v}}$ in (4.1) with (4.8), we have

$$
0=-\int_{\tau} \tilde{p} \nabla \cdot \tilde{\mathbf{v}} d x+\int_{\partial \tau} \tilde{\lambda} \tilde{\mathbf{v}} \cdot \nu_{\tau} d s=\int_{\tau} \tilde{p}^{2} d x+\int_{\partial \tau} \tilde{\lambda}^{2} d s
$$

therefore, $[\tilde{p}, \tilde{\lambda}]$ is zero.

The proof of the following lemma was suggested by Joseph Pasciak.

Lemma 4.3. Let $\hat{p}=[p, \lambda] \in W_{h}(\tau) \times \Lambda_{h}(\tau)$ and $\alpha_{\tau}=\alpha_{\mid \tau}$; then

$$
d_{\tau}(\widehat{p}, \widehat{p}) \simeq \alpha_{\tau}|\tau|^{1-2 / n} \sum_{\substack{\text { nodes : } \\ n_{i}, n_{j} \in \tau}}\left(\widehat{p}\left(n_{i}\right)-\widehat{p}\left(n_{j}\right)\right)^{2},
$$

with constants independent of the mesh parameter $h$ and $\alpha_{\tau}$.

Proof. Let $\tilde{\tau}$ denote the reference element, $W_{h}(\tilde{\tau})$ and $\mathbf{V}_{h}(\tilde{\tau})$ the reference spaces, and $F_{\tau}(\tilde{x})=b_{\tau}+B_{\tau} \tilde{x}$ the affine map mapping $\tilde{\tau}$ onto $\tau$ introduced in $\S 3$. For $[\tilde{q}, \tilde{\mu}] \in W_{h}(\tilde{\tau}) \times \Lambda_{h}(\tilde{\tau})$, define $\tilde{\nabla}_{h}[\tilde{q}, \tilde{\mu}] \in \mathbf{V}_{h}(\tilde{\tau})$ by

$$
\int_{\tilde{\tau}} \tilde{\nabla}_{h}[\tilde{q}, \tilde{\mu}] \cdot \tilde{\mathbf{v}} d x=-\int_{\tilde{\tau}} \tilde{q} \nabla \cdot \tilde{\mathbf{v}} d x+\int_{\partial \tilde{\tau}} \tilde{\mu} \widetilde{\mathbf{v}} \cdot \nu_{\tilde{\tau}} d s \quad \forall \widetilde{\mathbf{v}} \in \mathbf{V}_{h}(\tilde{\tau}) .
$$

Using Lemma 4.2, one has

$$
\int_{\tilde{\tau}} \widetilde{\nabla}_{h}[\tilde{q}, \tilde{\mu}] \cdot \widetilde{\nabla}_{h}[\tilde{q}, \tilde{\mu}] d x \simeq \sum_{\substack{\text { nodes : } \\ n_{i}, n_{j} \in \tilde{\tau}}}\left([\tilde{q}, \tilde{\mu}]\left(n_{i}\right)-[\tilde{q}, \tilde{\mu}]\left(n_{j}\right)\right)^{2},
$$

since both quadratic forms induce norms on $W_{h}(\tilde{\tau}) \times \Lambda_{h}(\tilde{\tau})$ modulo constant functions with equivalence constants depending only on the reference element and choice of mixed finite element space.

Letting $J_{\tau}=\left|\operatorname{det} B_{\tau}\right|$, define the following functions on the reference element:

$$
\tilde{p}=p \circ F, \quad \tilde{\lambda}=\lambda \circ F, \quad \tilde{\mathbf{u}}=\left(J_{\tau} B_{\tau}^{-1} \nabla_{h}^{A}[p, \lambda]\right) \circ F, \quad \tilde{A}=A \circ F .
$$

Under this change of variables for the mixed finite element spaces (cf. $[30,12])$, we have $\widetilde{\mathbf{u}} \in \mathbf{V}_{h}(\tilde{\tau})$ satisfying

$$
\int_{\tilde{\tau}} \frac{1}{J_{\tau}} B_{\tau}^{t} \tilde{A}^{-1} B_{\tau} \widetilde{\mathbf{u}} \cdot \widetilde{\mathbf{v}} d x=-\int_{\tilde{\tau}} \tilde{p} \nabla \cdot \widetilde{\mathbf{v}} d x+\int_{\partial \tilde{\tau}} \tilde{\lambda} \widetilde{\mathbf{v}} \cdot \nu_{\tilde{\tau}} d s \quad \forall \widetilde{\mathbf{v}} \in \mathbf{V}_{h}(\tilde{\tau})
$$

and

(4.16) $d_{\tau}(\widehat{p}, \widehat{p})=\int_{\tau} A^{-1} \nabla_{h}^{A}[p, \lambda] \cdot \nabla_{h}^{A}[p, \lambda] d x=\int_{\tilde{\tau}} \frac{1}{J_{\tau}} B_{\tau}^{t} \tilde{A}^{-1} B_{\tau} \tilde{\mathbf{u}} \cdot \tilde{\mathbf{u}} d x$.

Comparing (4.13) and (4.15), we see that

$$
\int_{\tilde{\tau}} \frac{1}{J_{\tau}} B_{\tau}^{t} \widetilde{A}^{-1} B_{\tau} \tilde{\mathbf{u}} \cdot \tilde{\mathbf{v}} d x=\int_{\tilde{\tau}} \widetilde{\nabla}_{h}[\tilde{p}, \tilde{\lambda}] \cdot \tilde{\mathbf{v}} d x \quad \forall \widetilde{\mathbf{v}} \in \mathbf{V}_{h}(\tilde{\tau})
$$

hence, 
(4.17) $\int_{\tilde{\tau}} \frac{1}{J_{\tau}} B_{\tau}^{t} \widetilde{A}^{-1} B_{\tau} \tilde{\mathbf{u}} \cdot \widetilde{\mathbf{u}} d x \leq C_{2} \alpha_{\tau} J_{\tau}\left\|B_{\tau}^{-1}\right\|^{2} \int_{\tilde{\tau}} \widetilde{\nabla}_{h}[\tilde{p}, \tilde{\lambda}] \cdot \widetilde{\nabla}_{h}[\tilde{p}, \tilde{\lambda}] d x$,

$$
\int_{\tilde{\tau}} \frac{1}{J_{\tau}} B_{\tau}^{t} \tilde{A}^{-1} B_{\tau} \tilde{\mathbf{u}} \cdot \widetilde{\mathbf{u}} d x \geq C_{1} \alpha_{\tau} J_{\tau}\left\|B_{\tau}\right\|^{-2} \int_{\tilde{\tau}} \widetilde{\nabla}_{h}[\tilde{p}, \tilde{\lambda}] \cdot \widetilde{\nabla}_{h}[\tilde{p}, \tilde{\lambda}] d x
$$

where $C_{1}$ and $C_{2}$ are defined in (2.3).

The proof of the lemma is completed by using (4.14), (4.16), (4.17), (4.18), and the following estimates, which hold uniformly for $\tau \in \mathscr{T}$, owing to the regularity of the mesh (cf. [13]):

$$
J_{\tau}=|\tau| /|\tilde{\tau}|, \quad\left\|B_{\tau}\right\| \leq C|\tau|^{1 / n}, \quad\left\|B_{\tau}^{-1}\right\| \leq C|\tau|^{-1 / n}
$$

\section{BALANCING DOMAIN DECOMPOSITION}

In this section, we reduce the dual problem (4.2) to a problem in terms of the variables on the subdomain boundaries using a standard nonoverlapping domain decomposition technique. We then consider the BDD method applied to this problem on the interfaces. That is, we study the iterative solution by conjugate gradients of the reduced problem preconditioned with the BDD preconditioner defined by Algorithm 1 below.

5.1. Reduction to an interface problem. We will need some additional notation related to the spaces of functions defined on the interfaces. With the domain $\Omega$ partitioned into nonoverlapping subdomains $\Omega_{i}$, we let $\mathscr{I}$ denote the set of internal interfaces

$$
\mathscr{F}=\bigcup_{i=1}^{k} \partial \Omega_{i} \backslash \partial \Omega \text {. }
$$

Let $\Lambda_{h}\left(\partial \Omega_{i}\right)$ denote the degrees of freedom associated with $\partial \Omega_{i} \backslash \partial \Omega$, and let $\Lambda_{h}(\mathscr{I})$ denote all the degrees of freedom on the subdomain interfaces. Define mappings $N_{i}: \Lambda_{h}\left(\partial \Omega_{i}\right) \rightarrow \Lambda_{h}(\mathscr{I})$ by extension by zero, i.e., for $\lambda_{i} \in \Lambda_{h}\left(\partial \Omega_{i}\right)$, define $N_{i} \lambda_{i} \in \Lambda_{h}(\mathscr{I})$ by

$$
N_{i} \lambda_{i}(x)= \begin{cases}\lambda_{i}(x) & \text { if } x \in \partial \Omega_{i}, \\ 0 & \text { otherwise. }\end{cases}
$$

Note that its adjoint $N_{i}^{T}: \Lambda_{h}(\mathscr{I}) \rightarrow \Lambda_{h}\left(\partial \Omega_{i}\right)$ is defined by $N_{i}^{T} \lambda=\lambda_{\mid \partial \Omega_{i} \backslash \partial \Omega}$.

Define bilinear forms $s_{i}: \Lambda_{h}\left(\partial \Omega_{i}\right) \times \Lambda_{h}\left(\partial \Omega_{i}\right) \rightarrow \mathbb{R}$ and corresponding linear maps $S_{i}: \Lambda_{h}\left(\partial \Omega_{i}\right) \rightarrow \Lambda_{h}\left(\partial \Omega_{i}\right)$ by

$$
s_{i}\left(\lambda_{i}, \mu_{i}\right)=\int_{j \Omega_{i} \backslash \partial \Omega}\left(S_{i} \lambda_{i}\right) \mu_{i} d s=d_{\Omega_{l}}\left(\widehat{p}_{i}\left(\lambda_{i}\right), \widehat{p}_{i}\left(\mu_{i}\right)\right),
$$

where $\widehat{p}_{i}\left(\lambda_{i}\right) \in W_{h}\left(\Omega_{i}\right) \times \Lambda_{h}\left(\Omega_{i}\right)$ satisfies

$$
\begin{aligned}
\widehat{p}_{i}\left(\lambda_{i}\right)=\lambda_{i} & \text { on } \partial \Omega_{i} \backslash \partial \Omega, \\
\widehat{p}_{i}\left(\lambda_{i}\right)=0 & \text { on } \partial \Omega_{i} \cap \partial \Omega, \\
d_{\Omega_{i}}\left(\widehat{p}_{i}\left(\lambda_{i}\right), \widehat{q}_{i}\right)=0 & \forall \widehat{q}_{i} \in W_{h}\left(\Omega_{i}\right) \times \Lambda_{h}^{0}\left(\Omega_{i}\right) .
\end{aligned}
$$


Define the bilinear form $s: \Lambda_{h}(\mathscr{I}) \times \Lambda_{h}(\mathscr{I}) \rightarrow \mathbb{R}$ and linear map $S:$ $\Lambda_{h}(\mathscr{I}) \rightarrow \Lambda_{h}(\mathscr{I})$ by

$$
s(\lambda, \mu)=\sum_{i=1}^{k} s_{i}\left(N_{i}^{T} \lambda, N_{i}^{T} \mu\right), \quad S=\sum_{i=1}^{k} N_{i} S_{i} N_{i}^{T} .
$$

The method of this paper solves a reduced problem $S \lambda=\bar{g}$ corresponding to the interface unknowns which, in variational form, may be written

$$
s(\lambda, \mu)=G(\mu) \text {. }
$$

The right-hand side is defined by

$$
G(\mu)=\sum_{i=1, \ldots, k} d_{\Omega_{i}}\left(\bar{p}_{i}, \widehat{p}_{i}\left(N_{i}^{T} \mu\right)\right),
$$

with $\widehat{p}_{i}\left(N_{i}^{T} \mu\right)$ defined as in (5.3)-(5.5) and $\bar{p}_{i}$ satisfying

$$
\begin{aligned}
\bar{p}_{i} & =0 \quad \text { on } \partial \Omega_{i} \backslash \partial \Omega, \\
\bar{p}_{i} & =g \quad \text { on } \partial \Omega_{i} \cap \partial \Omega, \\
d_{\Omega_{i}}\left(\bar{p}_{i},\left[q_{i}, \mu_{i}\right]\right) & =\int_{\Omega_{i}} f q_{i} d x \quad \forall\left[q_{i}, \mu_{i}\right] \in W_{h}\left(\Omega_{i}\right) \times \Lambda_{h}^{0}\left(\Omega_{i}\right) .
\end{aligned}
$$

The solution to the original problem is recovered by setting

$$
[p, \lambda]_{\mid \Omega_{i}}=\widehat{p}_{i}\left(\lambda_{i}\right)+\bar{p}_{i}, \quad \mathbf{u}=-\nabla_{h}^{A}[p, \lambda] .
$$

The interface bilinear form $s$ is symmetric and positive definite, cf. [16]. In fact, $S$ defined in (5.6) is nothing more than the Schur complement of the unknowns $\Lambda_{h}(\mathscr{I})$ in the matrix corresponding to the bilinear form $d$.

The maps $S_{i}$ are more easily evaluated by realizing that they are evaluation of the "Dirichlet-to-Neumann maps" for the mixed finite element method $[22,16]$ defined by

$$
S_{i} \lambda_{i}=-\mathbf{u}_{i} \cdot \nu
$$

where $\left\{\mathbf{u}_{i}, p_{i}\right\} \in \mathbf{V}_{h}\left(\Omega_{i}\right) \times W_{h}\left(\Omega_{i}\right)$ solves the Dirichlet problem

$$
\begin{gathered}
\int_{\Omega_{i}} A^{-1} \mathbf{u}_{i} \cdot \mathbf{v} d x-\int_{\Omega_{i}} p_{i} \nabla \cdot \mathbf{v} d x=-\int_{\partial \Omega_{i} \backslash \partial \Omega} \lambda_{i} \mathbf{v} \cdot \nu d s \quad \forall \mathbf{v} \in \mathbf{V}_{h}\left(\Omega_{i}\right) \\
\int_{\Omega_{i}} \nabla \cdot \mathbf{u}_{i} q=0 \quad \forall q \in W_{h}\left(\Omega_{i}\right) .
\end{gathered}
$$

In the algorithm presented below, we will also need to solve problems of the form $S_{i} \lambda_{i}=r_{i}$, or, in variational form,

$$
s_{i}\left(\lambda_{i}, \mu_{i}\right)=-\int_{\partial \Omega_{i} \backslash \partial \Omega} r_{i} \mu_{i} d s \quad \forall \mu_{i} \in \Lambda_{h}\left(\partial \Omega_{i}\right) .
$$

These correspond to evaluations of the "Neumann-to-Dirichlet maps" $r_{i} \mapsto \lambda_{i}$, which we implement by first solving the following Neumann problem for 
$\left\{\mathbf{u}_{i}, p_{i}\right\} \in \mathbf{V}_{h}\left(\Omega_{i}\right) \times W_{h}\left(\Omega_{i}\right)$ such that

$$
\begin{gathered}
\mathbf{u}_{i} \cdot \nu=r_{i} \quad \text { on } \partial \Omega_{i} \backslash \partial \Omega, \\
\int_{\Omega_{i}} \nabla \cdot \mathbf{u}_{i} q=0 \quad \forall q \in W_{h}\left(\Omega_{i}\right), \\
\int_{\Omega_{i}} A^{-1} \mathbf{u}_{i} \cdot \mathbf{v} d x-\int_{\Omega_{i}} p_{i} \nabla \cdot \mathbf{v} d x=0 \\
\forall \mathbf{v} \in \mathbf{V}_{h}\left(\Omega_{i}\right) \cap\left\{\mathbf{v} \cdot \nu=0 \text { on } \partial \Omega_{i} \cap \partial \Omega\right\},
\end{gathered}
$$

and then defining $\lambda_{i} \in \Lambda_{h}\left(\partial \Omega_{i}\right)$ by

$$
\int_{\partial \Omega_{i} \backslash \partial \Omega} \lambda_{i} \mu_{i} d s=-\int_{\Omega_{i}} A^{-1} \mathbf{u}_{i} \cdot \mathbf{v} d x+\int_{\Omega_{i}} p_{i} \nabla \cdot \mathbf{v} d x,
$$

where $\mathbf{v}$ is any element in $\mathbf{V}_{h}\left(\Omega_{i}\right)$ such that $\mathbf{v} \cdot \nu=\mu_{i}$. See [22] for details.

5.2. The BDD preconditioner. The BDD preconditioner for (5.7) makes use of a collection of weighting maps $D_{i}$ that form a decomposition of unity on $\Lambda_{h}(\mathscr{J})$, i.e.,

$$
\sum_{i=1}^{k} N_{i} D_{i} N_{i}^{T} \lambda=\lambda \quad \forall \lambda \in \Lambda_{h}(\mathscr{I}) .
$$

Additionally, it uses spaces $Z_{i} \subset \Lambda_{h}\left(\partial \Omega_{i}\right)$ such that

$$
\operatorname{Ker}\left(s_{i}\right) \equiv\left\{\lambda_{i} \in \Lambda_{h}\left(\partial \Omega_{i}\right) \mid s_{i}\left(\lambda_{i}, \mu_{i}\right)=0 \forall \mu_{i} \in \Lambda_{h}\left(\partial \Omega_{i}\right)\right\} \subseteq Z_{i}
$$

For the scalar second-order elliptic problems we consider in this paper, $\operatorname{Ker}\left(s_{i}\right)$ is empty if there is Dirichlet data imposed on any part of $\partial \Omega_{i} \cap \partial \Omega$; otherwise it is the set of functions that are constant on $\partial \Omega_{i}$. We note, however, that $Z_{i}$ may be any superset of the kernel. From the $Z_{i}$ 's, define a "coarse space" $\Lambda_{H}(\mathscr{I})$ by

$$
\Lambda_{H}(\mathscr{J})=\left\{\lambda \in \Lambda_{h}(\mathscr{I}) \mid \lambda=\sum_{i=1}^{k} N_{i} D_{i} \zeta_{i}, \quad \zeta_{i} \in Z_{i}\right\}
$$

We say that $\rho \in \Lambda_{h}(\mathscr{I})$ is balanced if $\rho$ is orthogonal to $\Lambda_{H}(\mathscr{I})$; that is,

$$
\int_{\mathscr{Y}} \rho \mu_{H} d s=0, \quad \forall \mu_{H} \in \Lambda_{H}(\mathscr{I}) \text {. }
$$

The process of replacing an unbalanced $\rho$ by a balanced $\rho^{\text {bal }}=\rho-$ $S \rho_{H}, \quad \rho_{H} \in \Lambda_{H}(\mathscr{I})$ will be called "balancing". Note that $\rho_{H}$ may be determined by solving a problem over the "coarse space" $\Lambda_{H}(\mathscr{F})$,

$$
s\left(\rho_{H}, \mu_{H}\right)=\int_{\mathscr{F}} \rho \mu_{H} d s \quad \forall \mu_{H} \in \Lambda_{H}(\mathscr{F}) .
$$

The action of the BDD preconditioner $M_{\text {bal }}$ is defined by Algorithm 1 .

Algorithm 1 (BDD Preconditioner). Given $\rho \in \Lambda_{h}(\mathscr{I})$, compute $M_{\text {bal }}^{-1} \rho$ as follows. Balance the original residual $\rho$, i.e., let $\rho_{H} \in \Lambda_{H}(\mathscr{I})$ be the solution 
to

$$
s\left(\rho_{H}, \mu_{H}\right)=\int_{\mathscr{I}} \rho \mu_{H} d s \quad \forall \mu_{H} \in \Lambda_{H}(\mathscr{I})
$$

and set

$$
\rho^{\mathrm{bal}}=\rho-S \rho_{H}
$$

Find any solutions $\lambda_{i} \in \Lambda_{h}\left(\partial \Omega_{i}\right)$ for each of the local problems

$$
s_{i}\left(\lambda_{i}, \mu_{i}\right)=\int_{\partial \Omega_{i} \backslash \partial \Omega} D_{i}^{T} N_{i}^{T} \rho^{\mathrm{bal}} \mu_{i} d s \quad \forall \mu_{i} \in \Lambda_{h}\left(\partial \Omega_{i}\right),
$$

and set

$$
\lambda=\sum_{i=1, \ldots, k} N_{i} D_{i} \lambda_{i}
$$

Balance the auxiliary problems by solving

$$
s\left(\lambda_{H}, \mu_{H}\right)=\int_{\mathscr{J}} \rho \mu_{H} d s-s\left(\lambda, \mu_{H}\right) \quad \forall \mu_{H} \in \Lambda_{H}(\mathscr{I}) .
$$

Set $M_{\mathrm{bal}}^{-1} \rho=\lambda+\lambda_{H}$.

In practice, the residual of the initial approximation should be balanced first as in (5.28); then the first balancing step (5.24) in every iteration can be omitted since the residual $\rho$ received from the conjugate gradients algorithm is already balanced.

In [24], it was proven that Algorithm 1 implements a well-defined operator that is symmetric and positive definite. The following abstract bound on the condition number of the preconditioned system follows from the proof of Theorem 3.2 in [24]. (The statement of the theorem in [24] is correct only in the case $Z_{i}=\operatorname{Ker}\left(s_{i}\right)$.)

Theorem 5.1. The map $M_{\mathrm{bal}}^{-1}: \Lambda_{h}(\mathscr{I}) \rightarrow \Lambda_{h}(\mathscr{I})$ defined in Algorithm 1 is symmetric, positive definite and is such that

$$
\operatorname{cond}\left(M_{\mathrm{bal}}, S\right)=\lambda_{\max }\left(M_{\mathrm{bal}}^{-1} S\right) / \lambda_{\min }\left(M_{\mathrm{bal}}^{-1} S\right) \leq \kappa,
$$

where

$$
\begin{gathered}
\kappa=\sup \left\{\frac{\sum_{j=1}^{k} s_{j}\left(N_{j}^{T} \sum_{i=1}^{k} N_{i} D_{i} \lambda_{i}, N_{j}^{T} \sum_{i=1}^{k} N_{i} D_{i} \lambda_{i}\right)}{\sum_{i=1}^{k} s_{i}\left(\lambda_{i}, \lambda_{i}\right)} \mid\right. \\
\lambda_{i} \in \Lambda_{h}\left(\partial \Omega_{i}\right) ; \int_{\partial \Omega_{i} \backslash \partial \Omega} \lambda_{i} \zeta_{i} d s=0, \forall \zeta_{i} \in \operatorname{Ker}\left(s_{i}\right) ; \\
\left.s_{i}\left(\lambda_{i}, \zeta_{i}\right)=0, \forall \zeta_{i} \in Z_{i}\right\} .
\end{gathered}
$$

The following lemma is a simple consequence of Theorem 3.3 in [25]. 
Lemma 5.2. For subdomain $\Omega_{i}$, define the weighting map $D_{i}$ as multiplication by edgewise (facewise in 3-D) constants,

$$
\left(D_{i} \lambda_{i}\right)(x)=\frac{\alpha_{i}}{\alpha_{i}+\alpha_{j}} \lambda_{i}(x), \quad x \in \partial \Omega_{i} \cap \partial \Omega_{j},
$$

and assume that there exists a number $R$ so that

$$
\frac{1}{\alpha_{j}} s_{j}\left(N_{j}^{T} N_{i} \lambda_{i}, N_{j}^{T} N_{i} \lambda_{i}\right) \leq \frac{1}{\alpha_{i}} R s_{i}\left(\lambda_{i}, \lambda_{i}\right)
$$

for all $i, j=1, \ldots, k$ and all $\lambda_{i} \in \Lambda_{h}\left(\partial \Omega_{i}\right)$ such that $\int_{\partial \Omega_{i} \backslash \partial \Omega} \lambda_{i} \zeta_{i} d s=0$, $\forall \zeta_{i} \in \operatorname{Ker}\left(s_{i}\right)$, and $s_{i}\left(\lambda_{i}, \zeta_{i}\right)=0, \forall \zeta_{i} \in Z_{i}$. Then there exists a constant $C$ not dependent on $h, H$ or $R$, so that $\kappa$ in Theorem 5.1 satisfies the bound

$$
\kappa \leq C R \text {. }
$$

\section{DeRIVATION OF THE CONDITION NUMBER ESTIMATE}

For a subset $\omega \subset \Omega$ composed of elements of $\mathscr{T}$, we will continue to associate with the pair $[p, \lambda] \in W_{h}(\omega) \times \Lambda_{h}(\omega)$ a function $\hat{p} \in \mathscr{P}(\omega)$ defined at the nodal points of the mixed finite element discretization. In particular, for a node $n_{i}$ of $\tau \subset \omega$,

$$
\widehat{p}\left(n_{i}\right)= \begin{cases}\lambda\left(n_{i}\right) & \text { if } n_{i} \in \partial \tau \\ p\left(n_{i}\right) & \text { otherwise }\end{cases}
$$

In Theorem 4.1 of $\S 4$, we have shown that the mixed finite element discretization of (1.1)-(1.2) over a region $\Omega_{i}$ gives rise to a quadratic form equivalent to

$$
\mathscr{Q}_{\Omega_{i}}(\hat{p}, \hat{p})=\alpha_{i} \sum_{\tau \in \mathscr{T}, \tau \subset \Omega_{i}}|\tau|^{1-2 / n} \sum_{\substack{\text { nodes: } \\ n_{i}, n_{j} \in \tau}}\left(\hat{p}\left(n_{i}\right)-\hat{p}\left(n_{j}\right)\right)^{2} .
$$

In this section, we will use this fact to show that

$$
s_{i}\left(\lambda_{i}, \lambda_{i}\right) \simeq \alpha_{i}\left|I_{h}^{\partial \Omega_{i}} \lambda_{i}\right|_{1 / 2, \partial \Omega_{i}}^{2},
$$

where $I_{h}^{\partial \Omega_{i}}$ is an interpolation operator defined below. Exploiting this equivalence, we will derive a bound on the condition number of the interface problem preconditioned by the BDD preconditioner similarly as in [25]. The key ingredient in both the definition of the interpolation operator and the subsequent proof of the equivalence is to relate the mixed finite element discretization to a conforming approximation on a related mesh.

6.1. A conforming equivalence. Given an element $\tau \in \mathscr{T}$, let $\widehat{\mathscr{T}}$ be a quasiregular subtriangulation of $\tau$ such that the vertices of the subtriangulation include the vertices of $\tau$ and the nodal points in $\tau$ pertaining to the degrees of freedom of $W_{h}(\tau) \times \Lambda_{h}(\tau)$. Note that such a subtriangulation need only be constructed on the reference element $\tilde{\tau}$ and then may be mapped to the individual elements. Several examples are given in Figures 1 and 2.

It will be useful to partition the vertices into two sets. A vertex of $\widehat{\mathscr{T}}_{\tau}$ will be called primary if it was a nodal point of $\tau$. Otherwise, we shall call the 

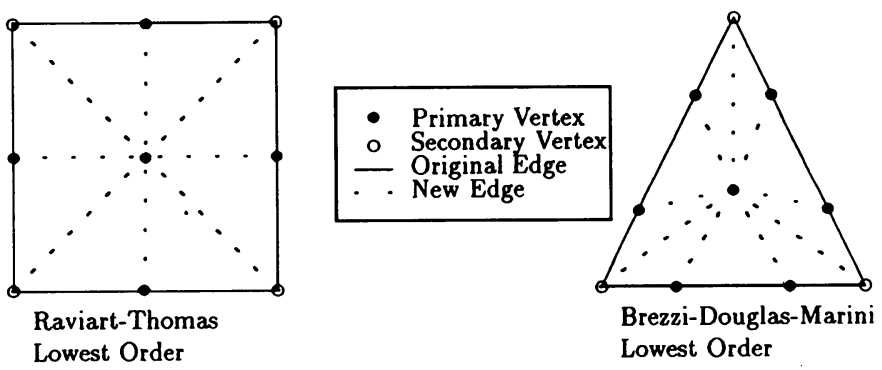

FIGURE 1. Examples of subtriangulations of two commonly used elements
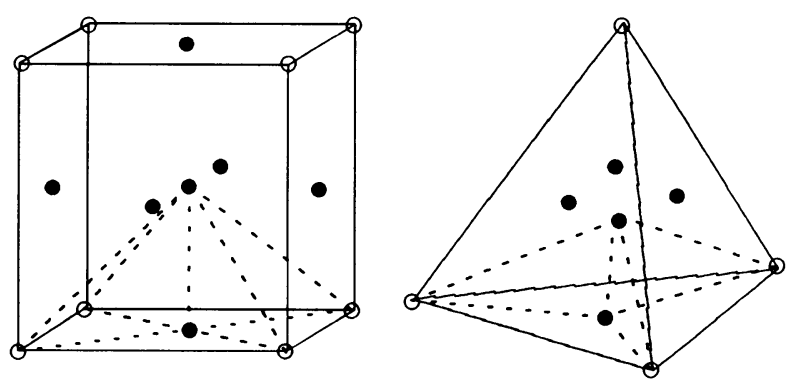

$$
\begin{array}{cl}
- & \text { Primary Vertex } \\
0 & \begin{array}{l}
\text { Secondary Vertex } \\
\text { Original Edge }
\end{array} \\
\ldots & \text { New Edge }
\end{array}
$$

FIGURE 2. Partial subtriangulation of the lowest-order Raviart-

Thomas-Nedelec elements

vertex secondary. The union of such subtriangulations gives rise to a refined triangulation of $\Omega$ which we denote by

$$
\widehat{\mathscr{T}} \equiv \bigcup_{\tau \in \mathscr{T}} \widehat{\mathscr{T}} .
$$

Using standard terminology, we will say that two vertices of the triangulation $\widehat{\mathscr{T}}$ are adjacent if there exists an edge of $\widehat{\mathscr{T}}$ connecting the vertices.

Let $U_{h}(\Omega)$ be the space of continuous piecewise linear functions subordinate to the triangulation $\widehat{\mathscr{T}}$. For a subdomain $\Omega_{i}$ define $U_{h}\left(\Omega_{i}\right)$ and $U_{h}\left(\partial \Omega_{i}\right)$ by restriction, i.e.,

$$
U_{h}\left(\Omega_{i}\right)=\left\{u_{\mid \Omega_{i}} \mid u \in U_{h}(\Omega)\right\}, \quad U_{h}\left(\partial \Omega_{i}\right)=\left\{u_{\mid \partial \Omega_{i}} \mid u \in U_{h}(\Omega)\right\} .
$$

Note that the functions in $U_{h}\left(\Omega_{i}\right)$ are naturally parameterized by the values they attain at the vertices. We may define a mapping $I_{h}^{\Omega_{i}}$ into $U_{h}\left(\Omega_{i}\right)$ for any 
function $\phi$ defined at the primary vertices contained in $\Omega_{i}$ by

$$
I_{h}^{\Omega_{i}} \phi(x)=\left\{\begin{array}{c}
\phi(x), \text { if } x \text { is a primary vertex; } \\
\text { the average of all adjacent primary vertices on the } \\
\text { boundary of } \Omega_{i}, \text { if } x \text { is a secondary vertex on the } \\
\text { boundary; } \\
\text { the average of all adjacent primary vertices, if } x \text { is a } \\
\text { secondary vertex in the interior of } \Omega_{i} ; \\
\text { the linear extension of vertex values, if } x \text { is not a vertex of } \widehat{\mathscr{T}} .
\end{array}\right.
$$

Since $I_{h}^{\Omega_{i}}$ is defined for any function defined at primary vertices, by an abuse of notation, we may understand $I_{h}^{\Omega_{i}}$ as a map from $W_{h}\left(\Omega_{i}\right) \times \Lambda_{h}\left(\Omega_{i}\right)$ into $U_{h}\left(\Omega_{i}\right)$, a map from $\mathscr{P}\left(\Omega_{i}\right)$ into $U_{h}\left(\Omega_{i}\right)$, and a map from $U_{h}\left(\Omega_{i}\right)$ into $U_{h}\left(\Omega_{i}\right)$.

Let $I_{h}^{\partial \Omega_{i}}$ be the mapping into $U_{h}\left(\partial \Omega_{i}\right)$ for functions defined on the primary vertices contained on the boundary of $\Omega_{i}$, by $I_{h}^{\partial \Omega_{i}} \lambda=\left(I_{h}^{\Omega_{i}} \widehat{p}\right)_{\mid \partial \Omega_{i}}$ where $\hat{p}$ is any element of $\mathscr{P}\left(\Omega_{i}\right)$ such that $\widehat{p}_{\mid \partial \Omega_{i}}=\lambda$. This map is well defined since the boundary values of elements in $I_{h}^{\Omega_{i}} \hat{p}$ depend only on the values of $\widehat{p}$ at the primary vertices on the boundary. Finally, let $\widetilde{U}_{h}\left(\Omega_{i}\right) \subset U_{h}\left(\Omega_{i}\right)$ and $\widetilde{U}_{h}\left(\partial \Omega_{i}\right) \subset U_{h}\left(\partial \Omega_{i}\right)$ be the range of $I_{h}^{\Omega_{i}}$ and $I_{h}^{\partial \Omega_{i}}$, respectively; that is,

$$
\tilde{U}_{h}\left(\boldsymbol{\Omega}_{i}\right)=\left\{\psi=I_{h}^{\Omega_{i}} \phi, \phi \in U_{h}\left(\boldsymbol{\Omega}_{i}\right)\right\}, \quad \tilde{U}_{h}\left(\partial \boldsymbol{\Omega}_{i}\right)=\left\{\psi_{\left.\left|\partial \Omega_{i}\right| \psi \in \tilde{U}_{h}\left(\boldsymbol{\Omega}_{i}\right)\right\} .}\right.
$$

Recall that for $\phi \in U_{h}\left(\Omega_{i}\right)$,

$$
\begin{aligned}
|\phi|_{1, \Omega_{i}}^{2} \simeq \sum_{\tau \in \widehat{J} \cdot \tau \subset \Omega_{i}}|\tau|^{1-2 / n} \sum_{\substack{\text { vertices: } \\
v_{i}, v_{j} \in \tau}}\left(\phi\left(v_{i}\right)-\phi\left(v_{j}\right)\right)^{2}, \\
\|\phi\|_{0, \Omega_{i}}^{2} \simeq \sum_{\tau \in \widehat{\mathcal{J}}, \tau \subset \Omega_{i}}|\tau| \sum_{\substack{\text { vertices : } \\
v_{i} \in \tau}} \phi\left(v_{i}\right)^{2} .
\end{aligned}
$$

Using these equivalences, we now show that the $I_{h}^{\Omega_{i}}$-projection is stable in $H^{1}\left(\Omega_{i}\right)$.

Lemma 6.1. There exists a constant $C>0$ independent of $h$ and $\left|\Omega_{i}\right|$ such that

$$
\begin{aligned}
\left|I_{h}^{\Omega_{i}} \phi\right|_{1, \Omega_{i}} & \leq C|\phi|_{1, \Omega_{i}} \quad \forall \phi \in U_{h}\left(\Omega_{i}\right), \\
\left\|I_{h}^{\Omega_{i}} \phi\right\|_{0, \Omega_{i}} & \leq C\|\phi\|_{0, \Omega_{i}} \quad \forall \phi \in U_{h}\left(\Omega_{i}\right) .
\end{aligned}
$$

Proof. We first consider the stability of the $H^{1}$-seminorm. Differences involving a secondary vertex and adjacent primary vertices can be bounded from differences involving the adjacent primary vertices as follows. If $v_{n+1}$ is a 
secondary vertex with adjacent primary vertices $v_{1}, \ldots, v_{n}$, and denoting $\phi_{j}=\phi\left(v_{j}\right)$, then

$$
\begin{aligned}
\left(\phi_{n+1}-\phi_{i}\right)^{2} & =\left(\frac{\sum_{j=1}^{n} \phi_{j}}{n}-\phi_{i}\right)^{2}=\frac{1}{n^{2}}\left(\sum_{j=1}^{n}\left(\phi_{j}-\phi_{i}\right)\right)^{2} \\
& \leq \frac{n}{n^{2}} \sum_{j=1}^{n}\left(\phi_{j}-\phi_{i}\right)^{2},
\end{aligned}
$$

by the Cauchy-Schwarz inequality. Since there is some maximum fixed number of secondary points per element, (6.4) now follows from (6.2). The stability in the $L^{2}$-norm is proven similarly using $(6.3)$ and the inequality $\phi_{n+1}^{2} \leq$ $\frac{1}{n} \sum_{i=1}^{n} \phi_{i}^{2}$, proved analogously to $(6.6)$.

6.2. Technical preliminaries. In this section two technical lemmas are proven that play an important role in the proof of the condition number bound. Lemma 6.2 demonstrates the equivalence between the $H^{1}$-norm of the discrete harmonic extension in the space $\widetilde{U}_{h}\left(\Omega_{i}\right)$ and the $H^{1 / 2}$-norm of the boundary values. Similar results for the conforming space $U_{h}\left(\Omega_{i}\right)$ are well known; see $[5,33]$, or [25]. A separate proof is required here since, in general, $\widetilde{U}_{h}\left(\boldsymbol{\Omega}_{i}\right) \neq U_{h}\left(\Omega_{i}\right)$.

Lemma 6.2. For $\widehat{\phi} \in \tilde{U}_{h}\left(\partial \Omega_{i}\right)$,

$$
\begin{gathered}
\inf _{\substack{\phi \in \tilde{U}_{h}\left(\Omega_{i}\right) \\
\phi \mid \partial \Omega}}\|\phi\|_{1, \Omega_{i}} \simeq\|\widehat{\phi}\|_{1 / 2, \partial \Omega_{i}}, \\
\inf _{\substack{\phi \in \widetilde{U}_{h}\left(\Omega_{j}\right) \\
\phi \mid \partial \Omega}}|\phi|_{1, \Omega_{i}} \simeq|\widehat{\phi}|_{1 / 2, \partial \Omega_{i} .} .
\end{gathered}
$$

Proof. By the standard Trace Theorem (see e.g. [26]), there exists a $C>0$ such that

$$
\|\widehat{\phi}\|_{1 / 2, \partial \Omega_{i}} \leq C\|\phi\|_{1, \Omega_{i}} \quad \forall \phi \in H^{1}\left(\Omega_{i}\right), \text { with } \phi_{\mid \partial \Omega_{i}}=\widehat{\phi} .
$$

Therefore, it is now enough to show that there exists a $C>0$ such that for all $\widehat{\phi} \in \widetilde{U}_{h}\left(\partial \Omega_{i}\right)$, there exists an extension $\phi^{E} \in \widetilde{U}_{h}\left(\Omega_{i}\right)$ satisfying $\phi_{\mid \partial \Omega_{i}}^{E}=\widehat{\phi}$ and

$$
\left\|\phi^{E}\right\|_{1, \Omega_{i}} \leq C\|\widehat{\phi}\|_{1 / 2, \partial \Omega_{i}} .
$$

By the extension theorem of Widlund [32], there exists a $C>0$ independent of $\hat{\phi}$ and $\phi \in U_{h}\left(\Omega_{i}\right)$ satisfying $\phi_{\mid \partial \Omega_{i}}=\widehat{\phi}$ and

$$
\|\phi\|_{1, \Omega_{i}} \leq C\|\widehat{\phi}\|_{1 / 2, \partial \Omega_{i}} .
$$

Taking $\phi^{E}=I_{h}^{\Omega_{i}} \phi$ and using the $H^{1}$-stability of $I_{h}^{\Omega_{i}}$ proven in Lemma 6.1, we have that

$$
\begin{aligned}
\left\|\phi^{E}\right\|_{1, \Omega_{i}} & \leq C\|\phi\|_{1, \Omega_{i}} \\
& \leq C\|\widehat{\phi}\|_{1 / 2, \partial \Omega_{i}},
\end{aligned}
$$

which proves (6.7). 
The equivalence of the seminorms follows from (6.7) and the equivalence of the $H^{1}\left(\Omega_{i}\right)$-norm to the $H^{1}\left(\Omega_{i}\right)$-seminorm and the $H^{\frac{1}{2}}\left(\partial \Omega_{i}\right)$-norm to the $H^{\frac{1}{2}}\left(\partial \Omega_{i}\right)$-seminorm on the spaces $H^{1}\left(\Omega_{i}\right) \cap\left\{\int_{\partial \Omega_{i}} \phi d s=0\right\}$ and $H^{\frac{1}{2}}\left(\partial \Omega_{i}\right) \cap$ $\left\{\int_{\partial \Omega_{i}} \phi d s=0\right\}$, respectively, cf. [25].

Lemma 6.3. There exists a constant $C>0$ independent of $h$ and $\left|\Omega_{i}\right|$ such that

$$
\left\|I_{h}^{\partial \Omega_{i}} \widehat{\phi}\right\|_{1 / 2, \partial \Omega_{i}} \leq C\|\widehat{\phi}\|_{1 / 2, \partial \Omega_{i}} \quad \forall \widehat{\phi} \in U_{h}\left(\partial \Omega_{i}\right) .
$$

Proof. Let $\phi \in U_{h}\left(\Omega_{i}\right)$ such that $\phi_{\mid \partial \Omega_{i}}=\widehat{\phi}$. By the standard Trace Theorem and Lemma 6.1, we have

$$
\left\|I_{h}^{\partial \Omega_{i}} \widehat{\phi}\right\|_{1 / 2, \partial \Omega_{i}} \leq C\left\|I_{h}^{\Omega_{i}} \phi\right\|_{1, \Omega_{i}} \leq C\|\phi\|_{1, \Omega_{i}} .
$$

The proof is completed by taking the infimum over the set of $\phi \in U_{h}\left(\Omega_{i}\right)$ such that $\phi_{\mid \partial \Omega_{i}}=\widehat{\phi}$ since for $\widehat{\phi} \in U_{h}\left(\partial \Omega_{i}\right)$,

$$
\inf _{\substack{\phi \in U_{h}\left(\Omega_{i}\right) \\ \phi \mid \partial \Omega=\hat{\phi}}}\|\phi\|_{1, \Omega_{i}} \simeq\|\widehat{\phi}\|_{1 / 2, \partial \Omega_{i}}
$$

see [32].

The following lemma is essentially Lemma 4.3 of [6] except that the support of $\phi_{j}$ is not wholly contained in $\partial \Omega_{i} \cap \partial \Omega_{j}$ but rather in a region overlapping neighboring faces of $\partial \Omega_{j}$ by $O(h)$.

Lemma 6.4. Let $\phi_{i} \in \widetilde{U}_{h}\left(\partial \Omega_{i}\right)$, and define $\phi_{j} \in \widetilde{U}_{h}\left(\partial \Omega_{j}\right)$ by

$$
\phi_{j}=I_{h}^{\partial \Omega_{j}} \phi, \quad \text { where } \phi= \begin{cases}\phi_{i} & \text { on } \partial \Omega_{i} \cap \partial \Omega_{j}, \\ 0 & \text { on } \partial \Omega_{j} \backslash \partial \Omega_{i} .\end{cases}
$$

Then there exists a $C>0$ independent of $H$ and $h$ such that

$$
\left\|\phi_{j}\right\|_{1 / 2, \partial \Omega_{j}}^{2} \leq C(1+\log (H / h))^{2}\left\|\phi_{i}\right\|_{1 / 2, \partial \Omega_{i}}^{2}
$$

Proof. By Lemma 4.3 of [6], we have

$$
\|\phi\|_{1 / 2, \partial \Omega_{j}}^{2} \leq C(1+\log (H / h))^{2}\left\|\phi_{i}\right\|_{1 / 2, \partial \Omega_{i}}^{2} .
$$

By Lemma 6.3,

$$
\left\|\phi_{j}\right\|_{1 / 2, \partial \Omega_{j}}^{2}=\left\|I_{h}^{\partial \Omega_{j}} \phi\right\|_{1 / 2, \partial \Omega_{j}}^{2} \leq C\|\phi\|_{1 / 2, \partial \Omega_{j}}^{2},
$$

and so the lemma is proven.

6.3. Condition number bound. The following theorem states the equivalence of the discrete seminorm defined by $s_{i}$ and the $H^{\frac{1}{2}}\left(\partial \Omega_{i}\right)$-seminorm of the boundary interpolant. A similar relation for the conforming spaces (without the need of an auxiliary interpolant) is the cornerstone of most substructuring domain decomposition theories; see, e.g., [5, 33]. 
Theorem 6.5. There exist constants $c, C>0$ independent of $h, H$ and $\alpha_{i}$ such that for all $\lambda_{i} \in \Lambda_{h}\left(\partial \Omega_{i}\right)$

$$
\alpha_{i} c\left|I_{h}^{\partial \Omega_{i}} \lambda_{i}\right|_{1 / 2, \partial \Omega_{i}}^{2} \leq s_{i}\left(\lambda_{i}, \lambda_{i}\right) \leq \alpha_{i} C\left|I_{h}^{\partial \Omega_{i}} \lambda_{i}\right|_{1 / 2, \partial \Omega_{i}}^{2}
$$

Proof. By a direct computation using definition (5.2),

$$
s_{i}\left(\lambda_{i}, \lambda_{i}\right)=\inf _{\substack{\hat{p} \in \mathscr{P}\left(\Omega_{i}\right) \\ \widehat{p} \mid \partial \Omega_{i}=\lambda_{i}}} d_{\Omega_{i}}(\widehat{p}, \hat{p}) .
$$

By Theorem 4.1 and (6.2),

$$
d_{\Omega_{i}}(\widehat{p}, \widehat{p}) \simeq \alpha_{i}\left|I_{h}^{\Omega_{i}} \widehat{p}\right|_{1, \Omega_{i}}^{2},
$$

where we bound terms involving secondary vertices as in the proof of Lemma 6.1 and terms involving the differences of primary vertices of $\tau \in \mathscr{T}$ by the sum of differences of nodes in $\widehat{\mathscr{T}}_{\tau}$. The lemma follows by taking the infimum of (6.17) over $\widehat{p}_{\mid \partial \Omega_{i}}=\lambda_{i}$ and using Lemma 6.2, since

$$
\begin{aligned}
s_{i}\left(\lambda_{i}, \lambda_{i}\right) & =\inf _{\substack{\hat{p} \in \mathscr{P}\left(\Omega_{i}\right) \\
\widehat{p} \mid \partial \Omega_{i}=\lambda_{i}}} d_{\Omega_{i}}(\widehat{p}, \hat{p}) \\
& \simeq \inf _{\substack{\hat{p} \in \mathscr{P}\left(\Omega_{i}\right) \\
\widehat{p} \mid \partial \Omega_{i}=\lambda_{i}}} \alpha_{i}\left|I_{h}^{\Omega_{i}} \widehat{p}\right|_{1, \Omega_{i}}^{2} \\
& \simeq \alpha_{i}\left|I_{h}^{\partial \Omega_{i}} \lambda_{i}\right|_{1 / 2, \partial \Omega}^{2} .
\end{aligned}
$$

Using the equivalence in the previous theorem, we may now prove a bound on the condition number of the preconditioned system.

Theorem 6.6. The interface operator $S$ preconditioned by the BDD preconditioner $M_{\text {bal }}$ defined in Algorithm 1 has a condition number $\kappa$ satisfying the bound

$$
\kappa \leq C(1+\log (H / h))^{2}
$$

in both two and three dimensions with the constant $C$ independent of $h, H$ and $\alpha_{i}$.

Proof. For our mixed spaces, there are no vertex degrees of freedom in two dimensions and no vertex or edge degrees of freedom in three dimensions. Hence, $N_{j}^{T} N_{i}=0$ unless $\Omega_{i}$ and $\Omega_{j}$ share an edge in two dimensions or a face in three dimensions. For $\lambda_{i} \in \Lambda_{h}\left(\partial \Omega_{i}\right), N_{j}^{T} N_{i} \lambda_{i} \in \Lambda_{h}\left(\partial \Omega_{j}\right)$ is defined by

$$
\left(N_{j}^{T} N_{i} \lambda_{i}\right)(x)= \begin{cases}\lambda_{i}(x) & \text { if } x \in \partial \Omega_{i} \cap \partial \Omega_{j}, \\ 0 & \text { otherwise. }\end{cases}
$$

By Theorem 6.5, we have

$$
s_{j}\left(N_{j}^{T} N_{i} \lambda_{i}, N_{j}^{T} N_{i} \lambda_{i}\right) \leq \alpha_{j} C\left|I_{h}^{\partial \Omega_{j}}\left(N_{j}^{T} N_{i} \lambda_{i}\right)\right|_{1 / 2, \partial \Omega_{j}}^{2} .
$$

Using Lemma 6.4, we see that

$$
\left|I_{h}^{\partial \Omega_{j}}\left(N_{j}^{T} N_{i} \lambda_{i}\right)\right|_{1 / 2, \partial \Omega_{j}}^{2} \leq C(1+\log (H / h))^{2}\left\|I_{h}^{j \Omega_{i}} \lambda_{i}\right\|_{1 / 2, \partial \Omega_{j}}^{2} .
$$


Hence, with another application of Theorem 6.5 and using the fact that $\|u\|_{1 / 2, \partial \Omega_{j}} \leq C|u|_{1 / 2, \partial \Omega_{j}}$ for all $u$ orthogonal to $Z_{j}$, cf. [25], we conclude that

$$
s_{j}\left(N_{j}^{T} N_{i} \lambda_{i}, N_{j}^{T} N_{i} \lambda_{i}\right) \leq C \frac{\alpha_{j}}{\alpha_{i}}(1+\log (H / h))^{2} s_{i}\left(\lambda_{i}, \lambda_{i}\right) .
$$

The proof is completed by appealing to the bound in Lemma 5.2.

\section{NUMERICAL EXPERIMENTS}

In this section we describe several numerical examples obtained using both a sequential and a parallel implementation of the BDD algorithm described in §5.2. The sequential implementation used the FORTRAN 77 code bdd available from MGNET by anonymous ftp to casper.cs.yale.edu in the directory /mgnet/jmandel. The code invokes user-supplied subroutines that implement the matrix-vector multiplications $S_{i} \lambda_{i}$ and solution of the possibly singular systems $S_{i} \lambda_{i}=r_{i}$. These subroutines were implemented by solving appropriate mixed problems in subdomains, cf. $\S 5.1$ of this paper.

The parallel implementation was written using the distributed memory, message-passing paradigm. The message-passing was implemented in PICL [19] to provide some degree of portability among message-passing architectures. The timing results presented here are from runs on the INTEL-Delta machine located at Caltech. In both the sequential and parallel implementations, existing sequential code was used to solve the elliptic problems on the subdomains.

In three experiments we consider the solution of the following elliptic problem on the domain $\Omega \subset \mathbb{R}^{3}$ with boundary $\partial \Omega=\partial \Omega_{D} \cup \partial \Omega_{N}$ :

$$
\begin{aligned}
-\nabla \cdot a(x) \nabla p & =f & & \text { in } \Omega, \\
p & =g_{D} & & \text { on } \partial \Omega_{D}, \\
-\nabla p \cdot \nu & =g_{N} & & \text { on } \partial \Omega_{N} .
\end{aligned}
$$

Equations (7.1)-(7.3) were discretized using finite differences which is the lowest-order Raviart-Thomas-Nedelec space defined on rectangular solids in 3D with quadrature [3i]. In all of our experiments, the mesh spacing in each coordinate direction was uniform and the spaces $Z_{i}$ in (5.20) were chosen as the constant functions on $\partial \Omega_{i}$ regardless of external boundary conditions. The initial guess of the solution was zero and iterations were continued until a reduction of $10^{-6}$ was achieved in the $l_{2}$-norm of the relative residual. The estimates of condition number were obtained by exploiting the similarity between conjugate gradients and Lanczos' method for finding eigenvalues using the code of Ashby, Manteuffel and Saylor [3].

The first two experiments were designed to test aspects of the condition number bound given in Theorem 6.6. The domain $\Omega$ was taken to be the unit cube with Dirichlet data imposed on the faces with normals parallel to the first coordinate axis. The functions $f, g_{D}, g_{N}$ were chosen such that

$$
p(x, y, z)=(\cosh (\pi(1-y))-\tanh (\pi) \sinh (\pi(1-y))) \cos (\pi x) .
$$


TABLE 1. Test Problem I, Laplace's equation

\begin{tabular}{||c|r|c||r|r||r|r||}
\hline & num. of & \multicolumn{1}{|c||}{ decompo- } & \multicolumn{2}{c||}{ BDD } & \multicolumn{2}{|c||}{ CG } \\
\cline { 4 - 7 }$h$ & domains & sition & cond. & iter. & cond. & inter. \\
\hline \hline $1 / 8$ & 8 & $2 \times 2 \times 2$ & 1.85 & 7 & 3.15 & 10 \\
$1 / 8$ & 64 & $4 \times 4 \times 4$ & 1.48 & 7 & 7.63 & 16 \\
$1 / 8$ & 512 & $8 \times 8 \times 8$ & 1.00 & 1 & 18.65 & 18 \\
$1 / 16$ & 8 & $2 \times 2 \times 2$ & 2.54 & 9 & 6.05 & 15 \\
$1 / 16$ & 64 & $4 \times 4 \times 4$ & 2.17 & 9 & 14.93 & 23 \\
$1 / 16$ & 512 & $8 \times 8 \times 8$ & 1.49 & 7 & 30.65 & 34 \\
$1 / 32$ & 8 & $2 \times 2 \times 2$ & 3.40 & 11 & 11.99 & 20 \\
$1 / 32$ & 64 & $4 \times 4 \times 4$ & 3.09 & 11 & 29.81 & 31 \\
$1 / 64$ & 64 & $4 \times 4 \times 4$ & 4.21 & 14 & 73.20 & 49 \\
\hline
\end{tabular}

TABLE 2. Test Problem II, discontinuous coefficient

\begin{tabular}{||c|r|r||r|r||r|r||}
\hline & \multicolumn{1}{|c||}{$\begin{array}{c}\text { num. of } \\
h\end{array}$} & \multicolumn{1}{c||}{ decompo- } & \multicolumn{2}{c||}{ BDD } & \multicolumn{2}{c||}{ CG } \\
\cline { 5 - 7 } & sition & cond. & iter. & cond. & inter. \\
\hline \hline $1 / 8$ & 64 & $4 \times 4 \times 4$ & 1.46 & 6 & 27.44 & 19 \\
$1 / 16$ & 64 & $4 \times 4 \times 4$ & 2.15 & 8 & 54.48 & 28 \\
$1 / 32$ & 64 & $4 \times 4 \times 4$ & 2.99 & 10 & 122.10 & 41 \\
$1 / 64$ & 64 & $4 \times 4 \times 4$ & 4.09 & 12 & 267.86 & 59 \\
\hline
\end{tabular}

Table 1 and Table 2 compare the performance of the nonpreconditioned and balanced preconditioned conjugate gradient method on the Schur complement system (i.e., the interface problem) in terms of condition number. Test Problem I is Laplace's equation with $a(x, y, z) \equiv 1$. Test Problem II has a coefficient that is piecewise constant on each subdomain and varies in magnitude from $10^{-48}$ to $10^{64}$. In particular

$$
a(x, y, z)= \begin{cases}10^{-i j k} & \text { if } i+j+k \text { is odd, } \\ 10^{i j k} & \text { if } i+j+k \text { is even, }\end{cases}
$$

where $i=\lfloor 1+4 x\rfloor, j=\lfloor 1+4 y\rfloor, k=\lfloor 1+4 z\rfloor$, and $\lfloor x\rfloor$ is the greatest integer not greater than $x$.

As illustrated in Figure 3 (next page), the use of the BDD preconditioner greatly reduces the condition number of the interface system. By analyzing the data in Table 1, we see that the interface system without preconditioning experiences a growth in the condition number like $1 / h$ as predicted by the theory in [16]. The condition number of the unpreconditioned system also grows with the number of subdomains, but not as strongly as $1 / H^{2}$ as predicted in [33]. Figure 4 (next page) shows that the condition number of the balanced preconditioned system grows like $\left(1+\log \frac{H}{h}\right)^{2}$. By comparing the results of the two tests, we observe that the convergence rate is independent of the jumps in the coefficients as the theory predicted. 


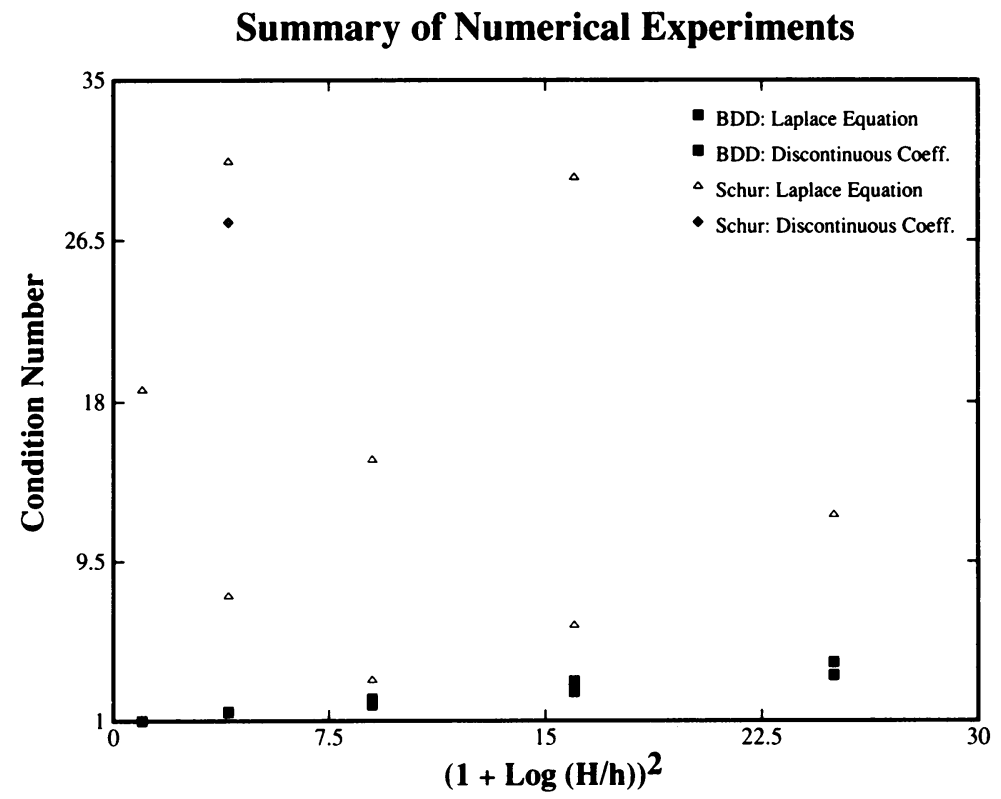

FIgURE 3. Scatter plot of results from Test Problems I and II

Summary of Numerical Experiments

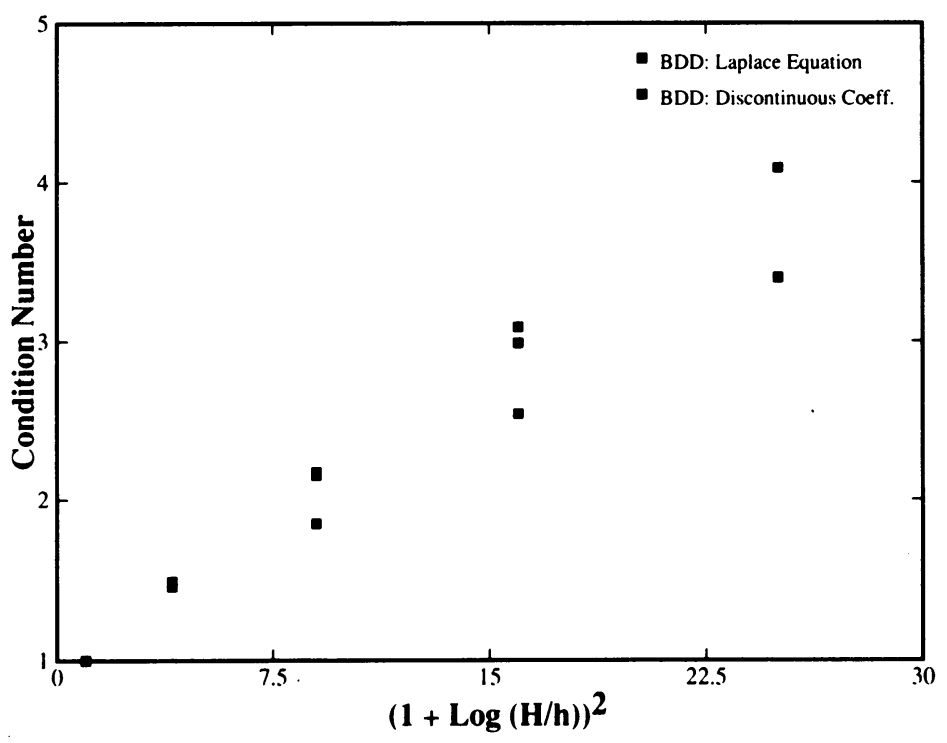

Figure 4. Scatter plot of results from Test Problems I and II: BDD results only 


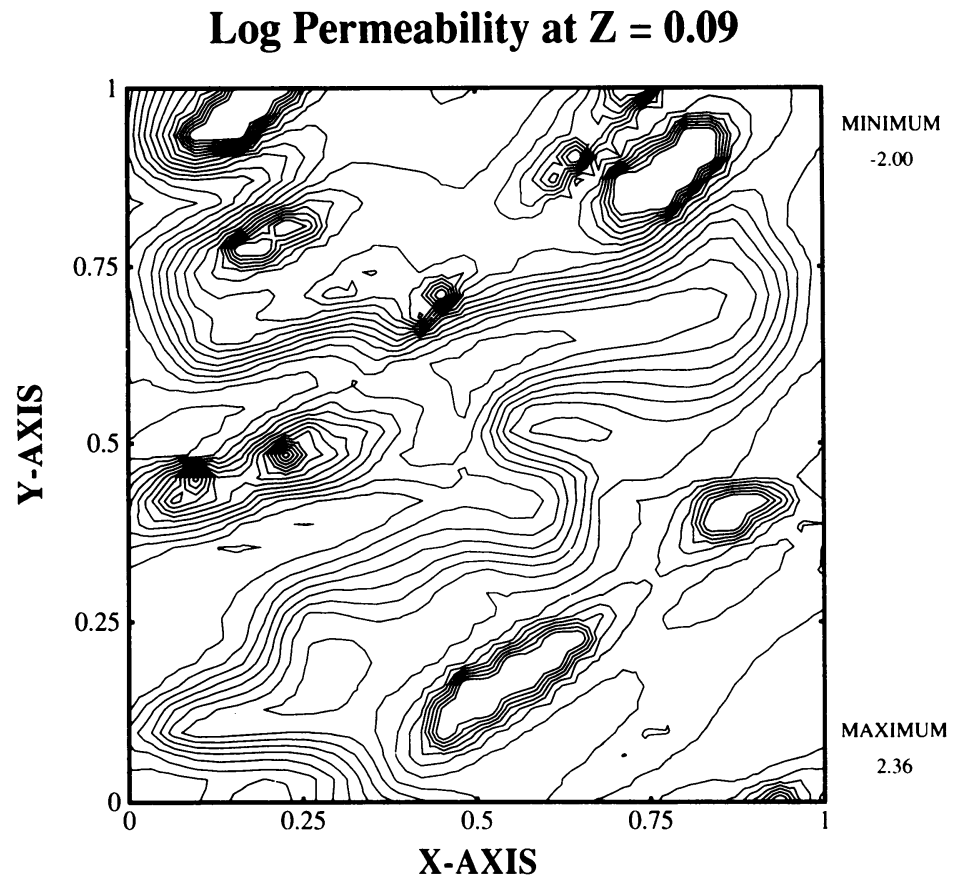

Figure 5. Coefficient of Test Problem III

To evaluate the parallel performance of the BDD algorithm, we consider a model "industrial-type" problem arising from the pressure equation in one time step of a miscible flow simulation with mobility ratio of 100 , see [29]. In this test problem, the domain was $\Omega=(0,1) \times(0,1) \times(0,1 / 8)$ and was discretized with a grid with $128 \times 64 \times 8$ elements. The aspect ratio is typical of porous media flow problems. The coefficient $a(x, y, z)$, a cross section of which is depicted in Figure 5, varies over five orders of magnitude in the entire domain. The number of unknowns in this problem is chosen to be relatively small to allow the problem to fit on a small number of processors. The run times are tabulated in Table 3 and depicted in Figure 6 (next page). Motivated by the work in [16], we used a generalization to the prescription of weighting functions in Lemma 5.2, choosing functions $D_{i}$ so that for $x \in \Omega_{i} \cap \Omega_{j}$

$$
\frac{D_{i}(x)}{D_{j}(x)}=\frac{\lim _{y \rightarrow x, y \in \Omega_{i}} a(y)}{\lim _{y \rightarrow x, y \in \Omega,} a(y)} .
$$

We note that the unpreconditioned interface problem did not converge in 200 iterations for any of the decompositions listed in Table 3 and had an estimated condition number in excess of $10^{5}$. 
Table 3. Test Problem III, miscible flow problem

\begin{tabular}{||c|c|r|r|r||}
\hline $\begin{array}{c}\text { Number of } \\
\text { Processors }\end{array}$ & $\begin{array}{c}\text { Decompo- } \\
\text { sition }\end{array}$ & $\begin{array}{c}\text { Run Time } \\
(\text { secs })\end{array}$ & \multicolumn{1}{c||}{$\begin{array}{c}\text { Cond. } \\
\text { Iter. }\end{array}$} \\
\hline \hline 16 & $8 \times 2 \times 1$ & 69.17 & 25 & 29.52 \\
16 & $4 \times 4 \times 1$ & 59.19 & 15 & 13.43 \\
16 & $4 \times 2 \times 2$ & 84.10 & 37 & 76.89 \\
32 & $8 \times 4 \times 1$ & 33.13 & 22 & 18.86 \\
32 & $4 \times 8 \times 1$ & 16.10 & 19 & 14.06 \\
32 & $4 \times 4 \times 2$ & 22.80 & 34 & 57.07 \\
32 & $2 \times 2 \times 8$ & 88.08 & 157 & 1532.49 \\
& & & & \\
64 & $8 \times 8 \times 1$ & 7.90 & 17 & 9.26 \\
64 & $8 \times 4 \times 2$ & 14.34 & 46 & 80.87 \\
64 & $4 \times 2 \times 8$ & 53.50 & 188 & 1749.38 \\
& & & & \\
128 & $16 \times 8 \times 1$ & 4.56 & 17 & 9.47 \\
128 & $8 \times 8 \times 2$ & 4.32 & 26 & 32.30 \\
256 & $16 \times 16 \times 1$ & 3.98 & 17 & 12.45 \\
256 & $16 \times 8 \times 2$ & 4.60 & 22 & 13.98 \\
\hline
\end{tabular}

Summary of Numerical Experiments

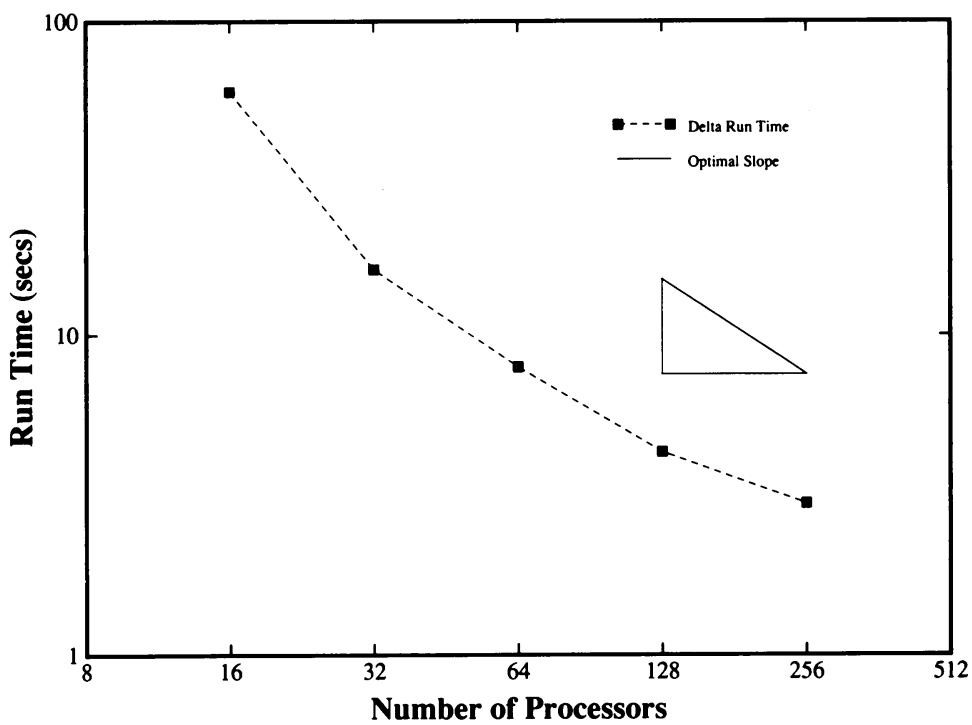

FIGURE 6. Scaling of the BDD algorithm 
Before addressing the performance of the parallel implementation, it is important to note that domain decomposition algorithms change with the decomposition chosen for the subdomains. For instance from the condition number estimates in Table 3, we see that the decompositions that give rise to subdomains with better aspect ratios perform better. As more processors are added, the dimension of coarse space $\Lambda_{H}(\mathscr{I})$ in (5.21) grows, as does the work required to solve the balancing problem (5.23). We see in Figure 6 the characteristic performance curve for domain decomposition algorithms. There is a region on a moderate number of processors where a faster than expected decrease in run time is observed, owing to the subdomain problems becoming easier to solve. Eventually, the subdomain problems become so small and the coarse problem becomes so large that the time for the coarse grid solve and message passing overhead dominate the run time. For our small test problem, we observe superlinear speed-up between 16 and 32 processors, near perfect linear speed-up between 32 and 64 processors, and degenerating speed-up for a larger number of processors.

\section{ACKNOWLEDGMENTS}

The authors appreciate access to the INTEL-Delta provided by the Center for Research on Parallel Computation. During the time of this research, Lawrence Cowsar and Mary Wheeler were supported by NSF grant DMS-9112847 and DOE grant DE-FG05-92ER25142, and Jan Mandel was supported by NSF grants DMS-9015259, ASC-9121431, and ASC-9217394.

\section{BIBLIOGRAPHY}

1. R. Adams, Sobolev spaces, Academic Press, New York, 1975.

2. D. N. Arnold and F. Brezzi, Mixed and nonconforming finite element methods: Implementation, postprocessing and error estimates, RAIRO Modél. Math. Anal. Numér. 19 (1985), 7-32.

3. S. F. Ashby, T. A. Manteuffel, and P. E. Saylor, A taxonomy for conjugate gradient methods, SIAM J. Numer. Anal. 27 (1990), 1542-1568.

4. J. F. Bourgat, R. Glowinski, P. Le Tallec, and M. Vidrascu, Variational formulation and algorithm for trace operator in domain decomposition calculations, Proc. Second Internat. Sympos. on Domain Decomposition Methods for Partial Differential Equations (T. Chan et al., eds.), SIAM, Philadelphia, PA, 1988, pp. 3-16.

5. J. H. Bramble, J. E. Pasciak, and A. H. Schatz, The construction of preconditioners for elliptic problems by substructuring. I, Math. Comp. 47 (1986), 103-134.

6. - The construction of preconditioners for elliptic problems by substructuring. IV, Math. Comp. 53 (1989), 1-24.

7. S. C. Brenner, A multigrid alorithm for the lowest order Raviart-Thomas mixed triangular finite element method, SIAM J. Numer. Anal. 29 (1992), 647-678.

8. F. Brezzi, On the existence, uniqueness and approximation of saddle point problems arising for Lagrangian multipliers, RAIRO Anal. Numér. 8 (1974), 129-151.

9. F. Brezzi, J. Douglas, Jr., R. Durán, and M. Fortin, Mixed finite elements for second order elliptic problems in three variables, Numer. Math. 51 (1987), 237-250. 
10. F. Brezzi, J. Douglas, Jr., M. Fortin, and L. D. Marini, Efficient rectangular mixed finite elements in two and three space variables, RAIRO Modél. Math. Anal. Numér. 21 (1987), 581-604.

11. F. Brezzi, J. Douglas, Jr., and L. D. Marini, Two families of mixed finite elements for second order elliptic problems, Numer. Math. 47 (1985), 217-235.

12. F. Brezzi and M. Fortin, Mixed and hybrid finite element methods, Springer Series in Comput. Math., vol. 15, Springer-Verlag, New York, 1991.

13. P. G. Ciarlet, The finite element method for elliptic problems, Stud. Math. Appl., vol. 4, North-Holland, New York, 1978.

14. L. C. Cowsar, Domain decomposition methods for nonconforming finite element spaces of Lagrange-type, Sixth Copper Mountain Conference on Multigrid Methods (N. D. Nelson, T. A. Manteuffel, and S. F. McCormick, eds.), NASA CP 3224, Hampton, VA, 1993, pp. 93-109.

15. — Dual-variable Schwarz methods for mixed finite elements, Numer. Math., submitted.

16. L. C. Cowsar and M. F. Wheeler, Parallel domain decomposition method for mixed finite elements for elliptic partial differential equations, in Glowinski et al. [21], pp. 358-372.

17. Y.-H. De Roeck and P. Le Tallec, Analysis and test of a local domain decomposition preconditioner, in Glowinski et al. [21], pp. 112-128.

18. L. J. Durlofsky and M. C. H. Chien, Development of a mixed finite-element based compositional reservoir simulator, Proceedings 12th SPE Symposium on Reservoir Simulation, SPE, Inc., 1993, Society of Petroleum Engineers, pp. 221-231.

19. G. A. Geist, M. T. Heath, B. W. Peyton, and P. H. Worley, A users' guide to PICL: $A$ portable instrumented communication library, Tech. Rep. ORNL/TM-11616, Oak Ridge National Laboratory, Aug. 1990.

20. R. Glowinski et al., eds., Proceedings of the First International Symposium on Domain Decomposition Methods for Partial Differential Equations, SIAM, Philadelphia, PA, Jan. 1987.

21. - eds., Proceedings of the Fourth International Symposium on Domain Decomposition Methods for Partial Differential Equations, SIAM, Philadelphia, PA, 1991.

22. R. Glowinski and M. F. Wheeler, Domain decomposition and mixed finite element methods for elliptic problems, in Glowinski et al. [20], pp. 144-172.

23. J. L. Lions and E. Magenes, Non-homogeneous boundary value problems and applications, Springer-Verlag, Berlin and New York, 1972.

24. J. Mandel, Balancing domain decomposition, Comm. Appl. Numer. Methods 9 (1993), 233241.

25. J. Mandel and M. Brezina, Balancing domain decomposition: theory and performance in two and three dimensions, Report No. 2, Center for Computational Mathematics, University of Colorado at Denver, 1993.

26. J. Necas, Les méthodes directes en théorie des équations elliptiques, Academia, Prague, 1967.

27. J. Nedelec, Mixed finite elements in $\mathbb{I}^{3}$, Numer. Math. 35 (1980), 315-341.

28. P. A. Raviart and J. M. Thomas, A mixed finite element method for second order elliptic problems, Mathematical Aspects of Finite Element Methods (I. Galligani and E. Magenes, eds.), Lecture Notes in Math., vol. 606, Springer-Verlag, Berlin, 1977, pp. 292-315.

29. T. F. Russell and M. F. Wheeler, Finite element and finite difference methods for continuous flows in porous media, Mathematics of Reservoir Simulation (R. E. Ewing, ed.), SIAM, Philadelphia, PA, 1983, ch. II, pp. 35-106.

30. J. M. Thomas, Sur l'analyse numérique des méthodes d'éléments finis hybrides et mixtes, thèse d'état, Université Pierre et Marie Curie, Paris, 1977.

31. A. Weiser and M. Wheeler, On convergence of block-centered finite differences for elliptic problems, SIAM J. Numer. Anal. 25 (1988), 351-357. 
32. O. B. Widlund, An extension theorem for finite element spaces with three applications, Numerical Techniques in Continuum Mechanics (W. Hackbusch and K. Witsch, eds.), GAMM, 1987, pp. 110-122.

33. - Iterative substructuring methods: Algorithms and theory for problems in the plane, in Glowinski et al. [20], pp. 113-128.

Department of Computational and Applied Mathematics, Rice University, Houston, TEXAS 77251-1892

Current address: AT\&T Bell Laboratories, Room 2C-302A, 600 Mountain Avenue, Murray Hill, New Jersey 07974

E-mail address: cowsar@research.att.com

Center for Computational Mathematics, University of Colorado at Denver, Denver, COLORADO 80217-3364

E-mail address: jmandel@colorado.edu

Department of Computational and Applied Mathematics, Rice University, Houston, TEXAS 77251-1892

E-mail address: mf w@rice.edu 